\title{
Epigenetic rejuvenation of the hippocampus by environmental enrichment
}

Sara Zocher ${ }^{1,2}$, Rupert W. Overall ${ }^{1,2}$, Mathias Lesche ${ }^{2,3}$, Andreas Dahl' ${ }^{2,3}$, Gerd Kempermann ${ }^{1,2 *}$

${ }^{1}$ German Center for Neurodegenerative Diseases (DZNE) Dresden, Tatzberg 41, 01307 Dresden,

${ }^{2}$ Center for Regenerative Therapies Dresden (CRTD), Technische Universität Dresden, Fetscherstraße 105, 01307 Dresden, Germany

${ }^{3}$ DRESDEN-concept Genome Center c/o Center for Molecular and Cellular Bioengineering

(CMCB), Technische Universität Dresden, Fetscherstraße 105, 01307 Dresden, Germany

*Corresponding author. E-mail: Gerd.Kempermann@dzne.de 


\section{Abstract}

The decline of brain function during aging is associated with epigenetic changes, including DNA

12 methylation. Lifestyle interventions can improve brain function during aging, but their influence

13 on age-related epigenetic changes is unknown. Using genome-wide DNA methylation sequencing,

14 we here show that environmental enrichment counteracted age-related DNA methylation changes

15 in the hippocampal dentate gyrus of mice. Specifically, environmental enrichment prevented the aging-induced $\mathrm{CpG}$ hypomethylation at target sites of the methyl-CpG-binding protein Mecp2, which is known to control neuronal functions. The genes at which environmental enrichment counteracted aging effects have described roles in neuronal plasticity, neuronal cell communication and adult hippocampal neurogenesis and are dysregulated with age-related cognitive decline in the human brain. Our results highlight the rejuvenating effects of environmental enrichment at the level of DNA methylation and give molecular insights into the specific aspects of brain aging that can be counteracted by lifestyle interventions. 


\section{Introduction}

Aging is associated with a progressive decline in brain function that manifests in cognitive impairments, increased risk for neurodegenerative diseases and loss of neural plasticity. Lifestyle factors, including physical exercise and cognitive stimulation, attenuate age-related reductions of brain function in humans ${ }^{1}$, contributing to what is called 'reserve' or 'maintenance' of brain function $^{2,3}$. In rodent models, environmental enrichment (ENR) promotes life-long brain plasticity and health ${ }^{4,5}$. ENR stimulates the function of existing neurons and adult neurogenesis in the hippocampus - a brain region with key roles in learning and memory, but high susceptibility to stress- and age-related impairments ${ }^{6}$. We set out to investigate the molecular mechanisms that link environmentally induced brain plasticity with improved brain maintenance during aging. Genome-wide DNA methylation changes are hallmarks of cellular aging ${ }^{5}$ and are used as biomarkers of the aging process ${ }^{6}$. In the hippocampus, the aging-induced dysregulation of the DNA methylation machinery is involved in the development of cognitive impairments ${ }^{9,10}$. Proper control of neuronal DNA methylation patterns in the adult brain is crucial for dynamic gene expression changes associated with synaptic plasticity and memory formation ${ }^{11-13}$. The sensitivity of neuronal methylomes to environmental stimuli, such as maternal behaviour ${ }^{14}$, learning stimuli ${ }^{15}$ or isolated neuronal activation ${ }^{16}$, contributes to the molecular mechanisms underlying experiencedependent brain plasticity ${ }^{17}$. Conversely, aberrant changes of neuronal DNA methylation patterns have been described in aging ${ }^{18,19}$ and in age-related disorders, such as Alzheimer's disease ${ }^{20,21}$. Since neuronal DNA methylation patterns are plastic and sensitive to environmental experiences, behavioral interventions could potentially rescue such aberrant DNA methylation changes and thereby promote brain health in old age. 
We therefore investigated the influence of life-long ENR on DNA methylation patterns in the hippocampal dentate gyrus and found that ENR counteracted aging-induced DNA methylation changes at genes related to neuronal plasticity and adult hippocampal neurogenesis. Our results highlight the potential of lifetime experiences to influence brain health in old age and provide a possible mechanism underlying the effects of lifestyle factors on brain aging.

\section{Environmental enrichment changes DNA methylation at brain plasticity genes in the adult}

\section{Results}

\section{dentate gyrus}

To first investigate whether ENR changes DNA methylation patterns in the adult dentate gyrus, female $\mathrm{C} 57 \mathrm{BL} / 6 \mathrm{JRj}$ mice were kept in ENR or standard housing cages (STD) for three months starting at an age of six weeks. Genome-wide DNA methylation profiling was performed on microdissected dentate gyrus tissue by reduced representation bisulfite sequencing (RRBS) ${ }^{22,23}$. No ENR-induced changes in global CpG methylation levels were detected (Fig. 1a), underscoring general genomic stability after ENR. Nevertheless, ENR modified methylation levels at 11,101 individual CpGs, i.e. $1.25 \%$ of all covered CpGs (Fig. 1b). Differentially methylated CpGs (dmCpGs) were depleted at $\mathrm{CpG}$ islands, $\mathrm{CpG}$ island shores, promoters and exons but enriched at enhancers, introns and intergenic regions of the genome (Fig. 1c). Additionally, ENR changed methylation at $0.019 \%$ of $\mathrm{CpHs}$ (in total only 750 differentially methylated $\mathrm{CpHs}$; dmCpHs), which showed a similar genomic distribution to dmCpGs but no enrichment at enhancers (Supplementary Fig. 1; Supplementary Data 1). Thus, ENR changed methylation in the dentate gyrus predominantly at $\mathrm{CpGs}$ located within regulatory genomic regions.

To explore the neuronal processes that are regulated by ENR, we performed gene set enrichment analysis with the 373 ENR-induced differentially methylated genes using expert-curated 
knowledge bases. Gene ontology $(\mathrm{GO})^{24}$ and Reactome pathway analyses ${ }^{25}$ showed that ENRinduced dmCpGs were enriched at genes involved in structural components of neurons, such as "axon part", "dendrite" and "dendritic spine" (Fig. 1d), and at genes with known functions in synaptic plasticity pathways, including glutamate receptor signaling and axon guidance (Fig. 1e; Supplementary Data 2). Enrichment analysis for genes from the Mammalian Adult Neurogenesis Gene Ontology (MANGO) ${ }^{26}$ highlighted that ENR changed DNA methylation at genes with described function in hippocampal neurogenesis, such as Fgfrl, Grial, Nfatc4, Ntf3, Flt1 and Thrb (Fig. 1f). In addition, enrichment analysis using the Synaptic Gene Ontologies (SynGO) knowledgebase $^{27}$ suggested that ENR-induced differentially methylated genes are enriched at

77 genes involved in synaptic assembly, organization of post-synapses and neurotransmitter signaling (Fig. 1g). These results indicated that ENR regulates pathways involved in neuronal plasticity and adult hippocampal neurogenesis in the murine dentate gyrus. 
a ENR does not affect global CpG methylation

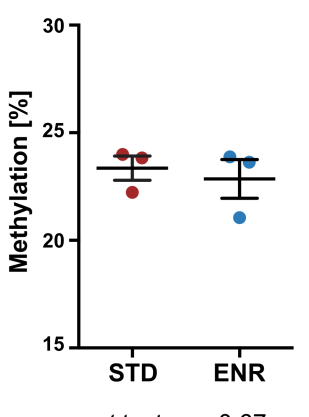

$t$-test: $p=0.67$ b ENR changes methylation at specific CpGs

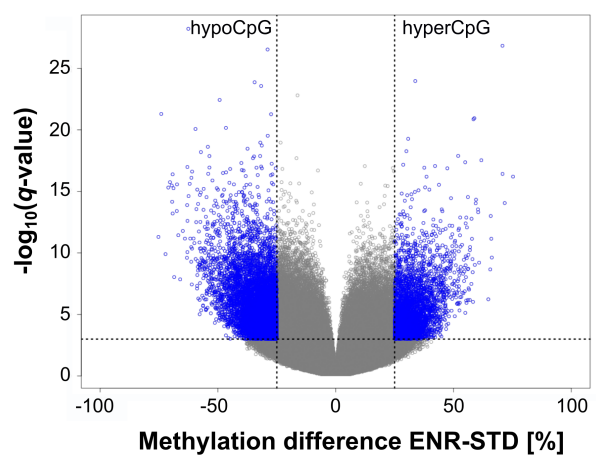

C Differentially methylated CpGs are enriched at gene regulatory elements

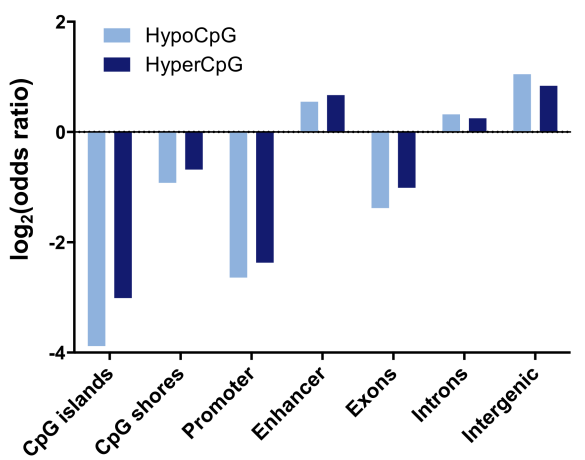

d GO enrichment of dmCpGs at genes involved in neurites and neuronal structure

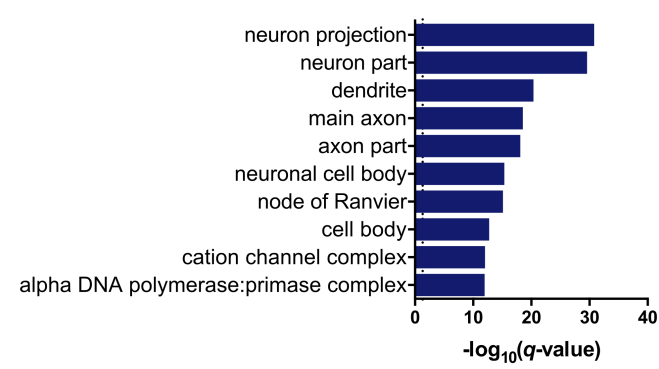

f Enrichment of genes involved in adult hippocampal neurogenesis (MANGO)

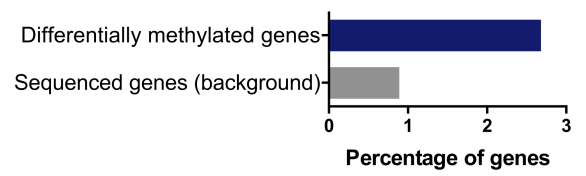

e Enrichment of dmCpGs in neuronal plasticity pathways

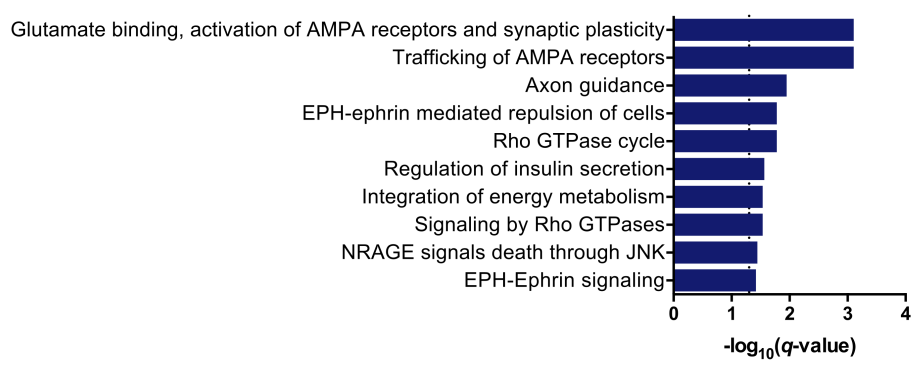

9 Enrichment of genes involved in organization of synapses (SynGO) neurotransmitter receptor localization to postsynaptic membrane regulation of neurotransmitter receptor localization structural constituent of postsynapse
synapse assembly \begin{tabular}{llllll}
\hline 0 & 1 & 2 & 3 & 4 & 5 \\
& $-\log _{10}(q$-value $)$
\end{tabular}

Fig. 1: Environmental enrichment changes CpG methylation in the adult dentate gyrus at genes with known role in neuronal plasticity, hippocampal neurogenesis and synapse organization. DNA methylation differences between 4.5-month-old mice housed in environmental enrichment (ENR) for three months and age-matched standard housed mice (STD) ( $n=3$ per group). a, ENR does not change global CpG methylation. b, Volcano plot highlighting ENR-induced differentially methylated CpGs (dmCpGs; methylation difference $>25 \%$ and $q<0.001$ ) in blue. Among the dmCpGs, $67.6 \%$ decreased methylation (hypoCpG) and $32.4 \%$ increased methylation in ENR (hyperCpG). c, Genomic distribution of dmCpGs. HypoCpG and hyperCpG are depleted at $\mathrm{CpG}$ islands, $\mathrm{CpG}$ island shores, promoters and exons $\left(\log _{2}\right.$ (odds ratio) $\left.<0\right)$ and enriched at enhancers, introns and intergenic regions $\left(\log _{2}(\right.$ odds ratio $)>0$ ). Adjusted $p$-values $<0.001$ for all regions. $\mathbf{d}$, Top ten significantly enriched cellular components from Gene Ontology (GO) enrichment analysis with differentially methylated genes (373 genes). e, ENR changed CpG methylation at genes involved in synaptic plasticity. Depicted are the top ten significantly enriched pathways from the Reactome database. $\mathbf{f}$, Genes functionally involved in hippocampal neurogenesis (as annotated in the MANGO database) are enriched among ENR-induced differentially methylated genes. Hypergeometric test: $p=0.0020$. g, Differentially methylated genes are involved in synapse organization and signaling. Depicted are significantly enriched biological processes from the SynGO database. The term "regulation of neurotransmitter receptor localization" was shortened from "regulation of neurotransmitter receptor localization to postsynaptic specialization membrane". Dashed lines in (d-e, g) indicate significance thresholds of $q<0.05$. 


\section{Age-related DNA methylation changes in the dentate gyrus}

101 To investigate the influence of ENR on age-related DNA methylation changes in the dentate gyrus,

102 RRBS was performed on dentate gyrus tissue from young (6.5-week-old) and aged (14-month-old)

103 mice, which had lived in STD or ENR for four days (young) or over a year (aged).

104 To determine age-related DNA methylation changes in the dentate gyrus, we compared DNA 105 methylation profiles between young STD mice and aged STD mice and detected 41,961 dmCpGs 106 and 6,600 dmCpHs (Supplementary Fig. 2a-b; Supplementary Data 3). While most dmCpGs were 107 hypomethylated in aged mice (77.93\% of dmCpGs), aging predominantly increased methylation 108 of $\mathrm{CpHs}(74.05 \%$ of $\mathrm{dmCpHs})$. The gene locations of aging-induced methylation changes in the 109 dentate gyrus were significantly enriched with genes previously reported to exhibit age-related 110 methylation changes in different tissues (Supplementary Fig. 2c). Additionally, more than $92.0 \%$

111 of the here identified age-related differentially methylated genes have been shown to change 112 methylation during aging in the mouse hippocampus by two independent studies ${ }^{18,28}$, highlighting 113 the robustness of aging-associated DNA methylation changes in the brain.

114 We found that age-related differentially methylated genes were significantly enriched in pathways 115 related to neuronal plasticity, neuronal signaling and energy metabolism (Supplementary Fig. 2d;

116 Supplementary Data 4). Remarkably, the highest enriched pathways of genes with age-related 117 DNA methylation changes overlapped considerably with the highest enriched pathways of ENR118 induced differentially methylated genes in the non-aged brain (compare Fig. 1e), which suggested 119 that aging and ENR changed DNA methylation at genes involved in similar pathways.

120 ENR counteracts age-related DNA methylation changes in the dentate gyrus

121 To investigate the effect of life-long ENR on aging, we first compared global aging-induced DNA 122 methylation changes between STD and ENR mice. In STD mice, aging was associated with a 
123 global $13.81 \%$ decrease in $\mathrm{CpG}$ methylation (Fig. 2a), which is in accordance with the

124 predominant $\mathrm{CpG}$ hypomethylation found at the individual $\mathrm{CpG}$ level (compare Supplementary

125 Fig. 2). In contrast, aged ENR mice did not show significant global CpG methylation differences

126 compared to young STD mice, suggesting that the age-related global CpG hypomethylation in the

127 dentate gyrus is at least partially prevented by ENR. No ENR-and age-related global methylation

128 changes were seen in the $\mathrm{CpH}$ context (Supplementary Fig. 3).

129 To analyze whether ENR counteracts aging-induced DNA methylation changes at specific genomic

130 loci, we determined cytosines where the methylation change induced by life-long ENR (difference

131 between aged ENR and aged STD mice) was opposite to the effect of aging (difference between

132 aged STD and young STD mice). From all CpGs that were hypomethylated with aging, $31.60 \%$

133 were significantly hypermethylated in aged ENR mice compared to aged STD mice (Fig. 2b).

134 Similarly, from all CpGs hypermethylated with aging, $32.17 \%$ were hypomethylated by ENR. In

135 the $\mathrm{CpH}$ context, $62.86 \%$ of aging-induced hypermethylated $\mathrm{CpHs}$ and $7.24 \%$ of hypomethylated

136 CpHs were changed by ENR in the opposite direction than by aging (Fig. 2b). In total, $31.73 \%$ of

137 all age-related $\mathrm{dmCpGs}$ and $48.42 \%$ of all aging-induced dmCpHs were counteracted by ENR.

138 Among those cytosines, the vast majority $(85.81 \%$ of CpGs; $98.47 \%$ of CpHs) did not show

139 differences between young and aged ENR mice, confirming that, in ENR mice, methylation at

140 these sites does not change with aging.

141 To compare magnitudes of methylation changes induced by aging and ENR, the absolute 142 methylation percentages of the $13,314 \mathrm{dmCpGs}$ and 3,196 dmCpHs at which ENR counteracted 143 aging effects were plotted (Fig. 2c-d). Compared to the young animals, the aged STD mice 144 exhibited a loss of highly methylated CpGs, which resulted in an accumulation of low or 145 unmethylated CpGs and $27 \%$ lower median $\mathrm{CpG}$ methylation levels. In contrast, the animals 146 housed in ENR for one year showed distributions and median CpG methylation percentages similar 
to young animals. Likewise, aged STD mice possessed different distributions and a $33 \%$ increase

in the median $\mathrm{CpH}$ methylation percentage compared to young animals (Fig. 2d-e), while aged

ENR mice were similar to young animals. These results suggest that ENR restores methylation at those age-sensitive $\mathrm{CpGs}$ and $\mathrm{CpHs}$ to the levels observed in young animals.

\section{a ENR counteracts age-dependent global CpG methylation changes}

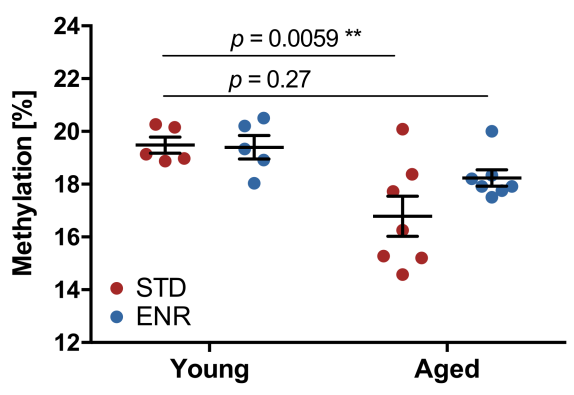

b ENR counteracts age-dependent methylation changes at $32 \%$ of $\mathrm{CpGs}$ and $48 \%$ of $\mathrm{CpHs}$
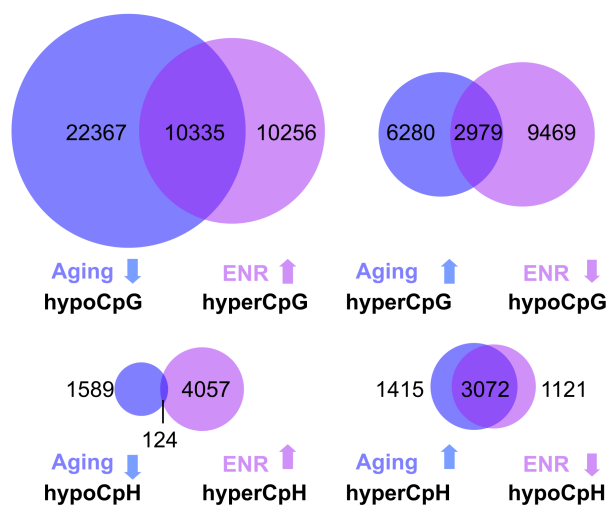
hyperCpG hypocpG

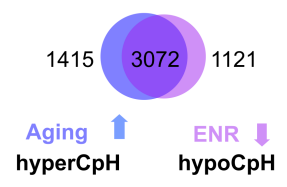

C ENR redistributes CpG and $\mathrm{CpH}$ methylation with aging
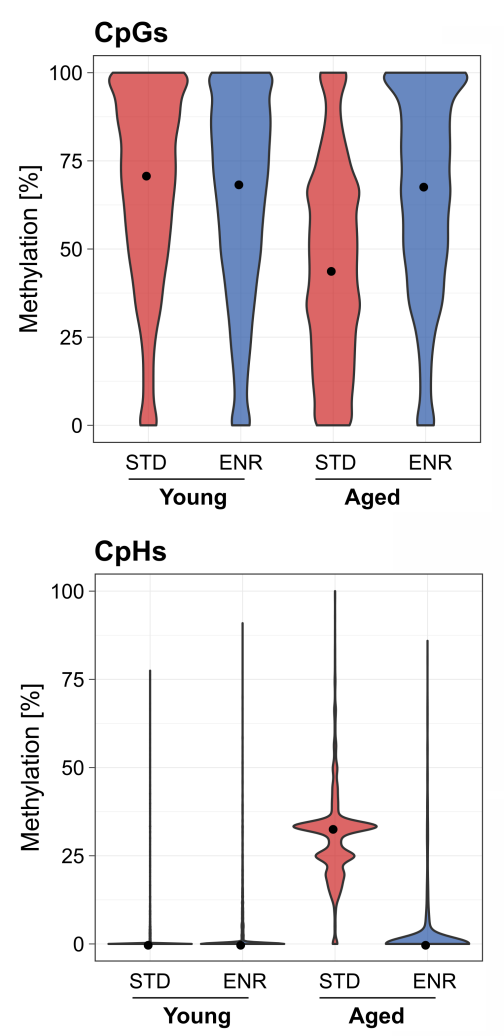

d ENR rejuvenates methylation at age-dependent CpGs and $\mathrm{CpHs}$

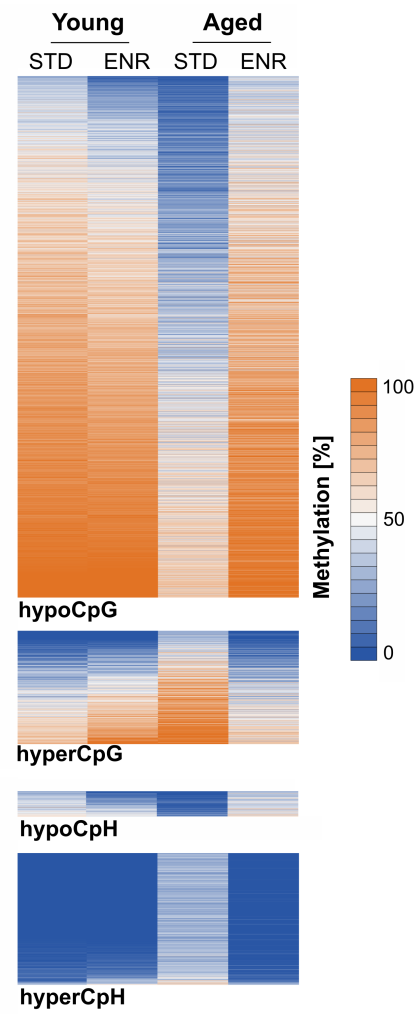

Fig. 2: Environmental enrichment protects the dentate gyrus from age-related $\mathrm{CpG}$ and $\mathrm{CpH}$ methylation changes. DNA methylation profiles of young mice housed in ENR or STD for four days (Young ENR; Young STD; $\mathrm{n}=5$ ) and aged mice housed in ENR or STD for one year (Aged ENR; Aged STD; $\mathrm{n}=7$ ). a, Aging decreases global CpG methylation in STD but not in ENR mice ( $p$-values from twoway ANOVA with Dunnett's post hoc test; depicted are means and s.e.m.). b, Aging and ENR induce opposite DNA methylation changes. Overlap of aging-induced hypoCpG with ENR-induced hyperCpG in aged mice (top left) and aging-induced hyperCpGs with ENR-induced hypoCpGs (top right). Overlap of aging- and ENR-induced $\mathrm{CpH}$ methylation changes (bottom). c, Age- and ENR-dependent changes of the distributions of methylation percentages of the 13,314 CpGs and 3,196 CpHs counteracted by ENR. Medians of methylation percentages per group are highlighted as black dots (CpGs; Young STD: $71.05 \%$, Young ENR: $68.57 \%$, Aged STD: $44.03 \%$, Aged ENR: $67.93 \%$. CpHs; Young-STD: $0 \%$, Young-ENR: $0 \%$, Aged-STD: $32.85 \%$, Aged ENR: $0 \%$ ). d, Heatmaps depicting the absolute methylation percentages in the individual groups at individual $\mathrm{CpGs}$ and $\mathrm{CpHs}$. While aged STD mice show distinct methylation at individual sites, aged ENR mice show methylation levels similar to young animals. 
166 To increase temporal resolution of age-related DNA methylation changes, we integrated the data 167 from the independent first experiment with 4.5-month-old (hereafter referred to as middle-aged)

168 mice housed in ENR or STD for three months (compare Fig. 1). The CpG and CpH methylation 169 levels of middle-aged mice were similar to young animals and did not show the age-related loss of 170 CpG methylation observed in aged STD mice (Supplementary Fig. 4a). Further comparison of the 171 effects of life-long ENR with age-related changes that occured from middle-aged STD to aged STD 172 mice showed that the pattern of $\mathrm{dmCpGs}$ and $\mathrm{dmCpHs}$ that were counteracted by ENR was similar 173 to that observed for young animals (Supplementary Fig. 4b). Additionally, 43.71\% of CpGs and $17456.32 \%$ of $\mathrm{CpHs}$ at which ENR counteracted aging were also differentially methylated between 175 middle-aged STD mice and aged STD mice. This suggests that age-related epigenetic changes 176 became pronounced only after an age of 4.5 months and that the environmental influence on age177 related methylation changes is itself age-dependent.

\section{Genomic distribution of environmentally sensitive age-related DNA methylation changes}

179 To further characterize the influence of ENR on aging-induced epigenetic reconfigurations, we 180 analyzed the genomic distribution of $\mathrm{CpGs}$ and $\mathrm{CpHs}$ at which ENR counteracted age-related 181 methylation changes. Both dmCpGs and $\mathrm{dmCpHs}$ were distributed over all chromosomes in the 182 genome (Fig. 3a), with dmCpGs depleted at exons, $\mathrm{CpG}$ islands, $\mathrm{CpG}$ island shores, promoters and 183 super-enhancers, but significantly enriched at introns, inter-genic regions and enhancers (Fig. 3b).

184 The distribution of $\mathrm{dmCpHs}$ over genomic features was similar to dmCpGs, but they were not 185 enriched at enhancers. Because DNA methylation of enhancers is particularly involved in the 186 regulation of gene expression by interaction with transcription factors ${ }^{29}$, we performed a 187 transcription factor motif analysis of the $1,472 \mathrm{dmCpGs}$ located within enhancers. The only 188 significantly enriched transcription factor was Mecp2 (Fig. 3c), which binds methylated cytosines 
via its methyl-CpG-binding domain ${ }^{30}$. Mecp2 was also the strongest enriched transcription factor

190 when motif enrichment analysis was performed on all 13,314 dmCpGs (independent of genomic

191 location), which suggested that Mecp2 binding occurs genome-wide and is not restricted to

192 dmCpGs located within enhancers (Supplementary Data 5). In contrast to dmCpGs, no enrichment

193 of Mecp2 motifs was found at dmCpHs (Fig. 3c).

194 To validate the enrichment of Mecp2 binding sites at dmCpGs, we used two existing Mecp2 ChIP195 sequencing datasets derived from adult mouse cortex and prefrontal cortex, respectively ${ }^{31}$. Similar 196 to Mecp2 motifs, ChIP-derived Mecp2-bound genomic regions were significantly enriched at 197 dmCpGs (hypergeometric tests; cortex: $p<1 \mathrm{E}-300$; prefrontal cortex: $p=1.4 \mathrm{E}-286$ ).

198 Because Mecp2 is known to specifically bind methylated cytosines, we analyzed the direction of 199 the methylation change of Mecp2 target dmCpGs with aging and ENR. The vast majority of Mecp2 $200 \quad$ targets $(87.31 \%)$ were hypomethylated with aging and hypermethylated by ENR (Fig. 3d). Aging201 induced hypomethylated CpGs were significantly enriched among the Mecp2 targets $(p=2.7 \mathrm{E}-$ 202 36). These results suggest that ENR prevents the age-associated CpG hypomethylation of Mecp2 203 binding sites and, thereby, facilitates Mecp2 binding in the aged dentate gyrus.

204 Annotation of $\mathrm{dmCpGs}$ and $\mathrm{dmCpHs}$ to their associated gene targets identified 676 genes at which 205 ENR counteracted age-related methylation changes. Two thirds of these genes were protein-coding 206 genes, but also lncRNA and minor percentage of ncRNAs (such as microRNAs) and pseudogenes 207 changed DNA methylation (Fig. 3e). The differentially methylated genes were significantly 208 enriched with genes that are known to be transcriptionally repressed by Mecp2 $2^{31}$ (Fig. 3f), further 209 supporting that ENR counteracts age-related DNA methylation changes at Mecp2 targets. 
a Chromosomal locations of differentially methylated cytosines

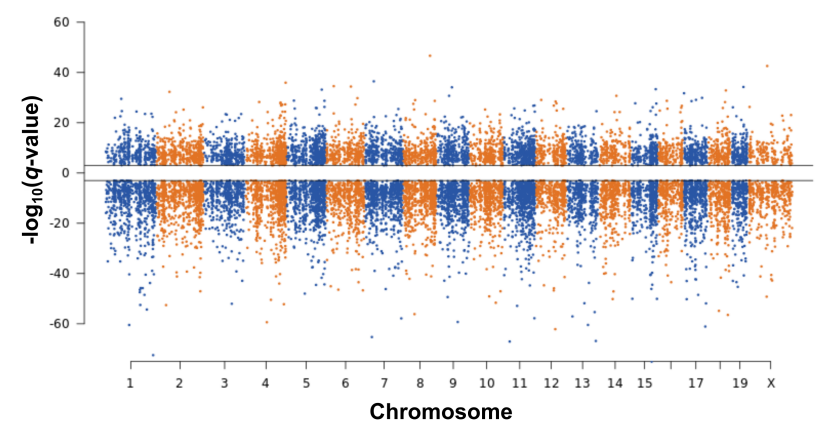

C Enrichment of Mecp2 motifs at dmCpGs

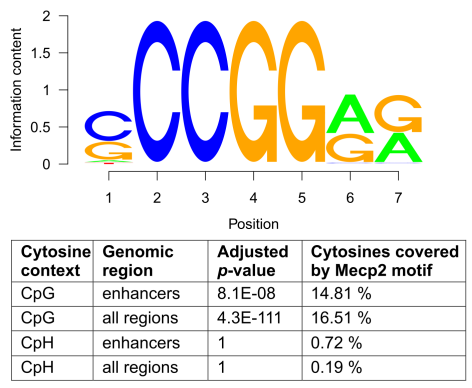

ENR counteracts hypomethylation of Mecp2 targets

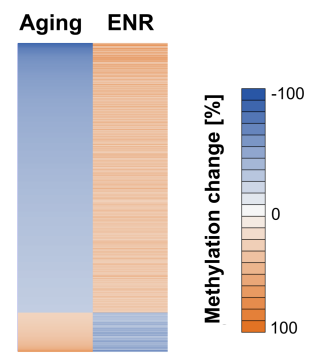

hypoCpGs: $p=2.7 \mathrm{E}-36$ b Enrichment of dmCpGs at regulatory regions

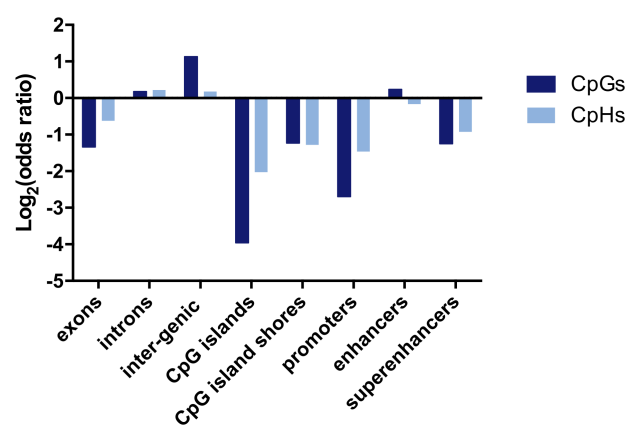

e Gene types affected by dmCpGs and dmCpHs

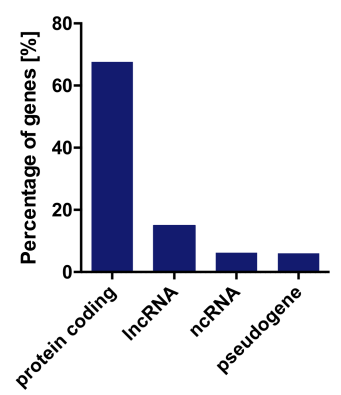

Fig. 3: Genomic distribution of cytosines at which ENR counteracts age-related methylation changes. a, Manhattan plot depicting the distribution of $\mathrm{dmCpGs}$ and $\mathrm{dmCpHs}$ over chromosomes. $Q$-values indicate the significance of the aging-induced methylation changes with the sign corresponding to the direction of the change (negative $-\log _{10}\left(q\right.$-value) for hypomethylated cytosines; positive $-\log _{10}(q$-value $)$ for hypermethylated cytosines). b, $\mathrm{CpGs}$ and $\mathrm{CpHs}$ are depleted at $\mathrm{CpG}$ islands, $\mathrm{CpG}$ island shores, promoters and exons $\left(\log _{2}\right.$ (odds ratio) $\left.<0\right)$, but enriched at introns and inter-genic regions of the genome $\left(\log _{2}(\right.$ odds ratio) $>0$ ). Additionally, $\mathrm{CpGs}$ but not $\mathrm{CpHs}$ are enriched at enhancer regions. Adjusted $p<0.001$ for all regions, except $\mathrm{CpHs}$ at enhancers (adjusted $p=0.061$ ). c, Transcription factor binding analysis revealed enrichment of Mecp2 motifs at dmCpGs located within enhancers (1,472 CpGs) as well as at all regulated $13,314 \mathrm{dmCpGs}$ (independent of genomic location). Mecp2 motifs were not enriched at dmCpHs. Depicted are the Mecp2 motif as annotated in the database motifDb and the results of the enrichment analysis. d, The 2,198 dmCpGs that fall into Mecp2 targets sites are enriched with CpGs that were hypomethylated with aging ( $p$-value from hypergeometric test compared to all 13,314 CpGs). e, Classification of gene targets of cytosines at which ENR counteracts aging-induced methylation changes (676 differentially methylated genes). f, Previously identified Mecp2-repressed genes ${ }^{32}$ are enriched among the differentially methylated genes ( $p$-value from hypergeometric test).

Experience protects neuronal plasticity and neurogenesis-related genes from age-related

\section{DNA methylation changes}

To identify aspects of brain aging that are sensitive to environmental stimulation, we performed

GO and pathway enrichment analyses of genes at which ENR counteracted aging-induced 
232 methylation changes and generated an integrated map based on gene overlaps between enriched 233 terms ${ }^{33}$. We identified three main clusters which were related to neuronal plasticity, cell 234 communication and developmental programs (Fig. 4a; Supplementary Data 6). Among those, the 235 "neuron plasticity" cluster contained the highest number of enriched terms. It comprised pathways 236 related to structural plasticity of neurons, including axon guidance and synapse organization, as 237 well as processes involved in neuronal activation, such as synaptic signaling and regulation of 238 potassium channels. In the "cell communication" cluster, we found signaling pathways that involve 239 secreted molecules, such as insulin and growth factors, and a group of enriched terms related to 240 cell adhesion, including formation of cellular junctions by cadherins, adhesion to extracellular 241 matrix and extracellular matrix remodeling by regulation of collagen formation. The third cluster 242 contained pathways involved in organ morphogenesis but also pathways related to nervous system 243 development, including neuronal fate specification and precursor proliferation.

244 To correlate differential cytosine methylation with cellular changes in the brain, we investigated 245 adult hippocampal neurogenesis which has a role in cognitive flexibility and decreases with 246 aging $^{34}$. We found that genes at which ENR counteracted age-related methylation changes were 247 significantly enriched for genes functionally involved in adult hippocampal neurogenesis as 248 annotated in MANGO (Fig. 4b). Accordingly, mice housed in ENR for one year showed a $60 \%$ 249 increase in the number of new-born neurons in the dentate gyrus compared to aged STD mice 250 (Fig. 4c-d), suggesting that continuous ENR increases hippocampal neurogenesis throughout the 251 lifespan. In contrast, only trends towards increased precursor proliferation and no change in total 252 precursor cell numbers were observed in aged ENR compared to aged STD mice (Supplementary 253 Fig. 5). This suggests that life-long ENR stimulates new-born neurons during an immature phase 254 but has only minor effects on neural precursor cells, which is in accordance with what has been 255 observed after shorter periods of ENR in young adult and aged mice ${ }^{34}$. 
256 To analyze whether ENR protects from aging effects by actively changing DNA methylation 257 throughout the lifespan, we overlapped genes where ENR counteracted DNA methylation changes 258 in aged mice with DNA methylation changes induced by shorter periods of ENR in non-aged mice. 259 In total, $24.02 \%$ of genes that changed DNA methylation after four days of ENR in young mice 260 and $30.03 \%$ of genes that changed DNA methylation after three months of ENR in middle-aged 261 mice overlapped with genes at which ENR counteracted age-related changes (Supplementary 262 Fig. 6a). Additionally, ENR-induced differentially methylated genes were significantly enriched 263 with genes that changed transcription after acute neuronal activation in the dentate gyrus ${ }^{35}$ 264 (Supplementary Fig. 6b). These results might suggest that active ENR-induced DNA methylation 265 changes at neuronal activity regulated genes counteract the development of age-related DNA 266 methylation changes. 
a Functional map of ENR versus aging interactions in the dentate gyrus
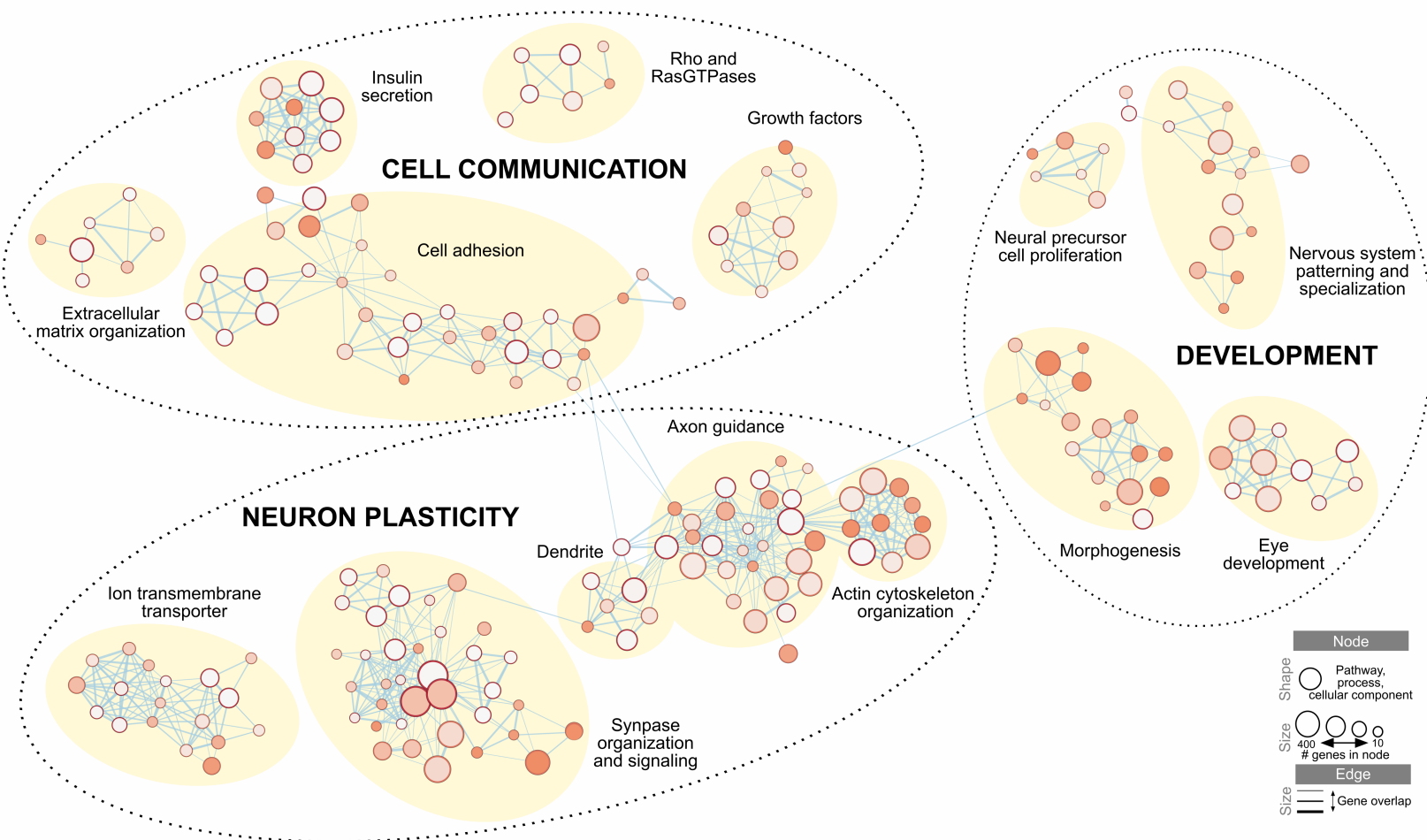

DEVELOPMENT

Morphogenesis
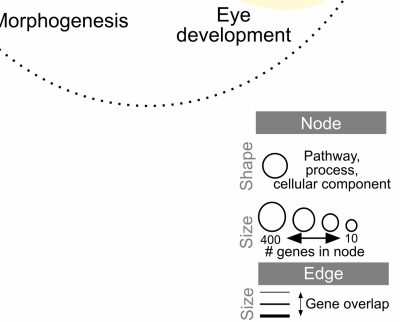

b ENR counteracts aging effects at genes involved in hippocampal neurogenesis (MANGO)

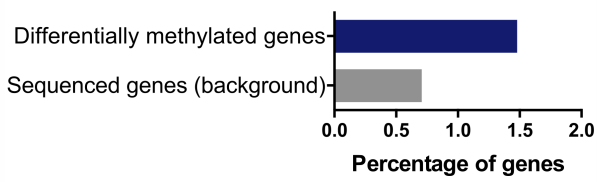

Fgf1, Fgfr2, Ntf3, Flt1, Cit, Ephb1, Nos1, Pax6, Dyrk1a, Glp1r

C ENR increases hippocampal d neurogenesis in aged mice
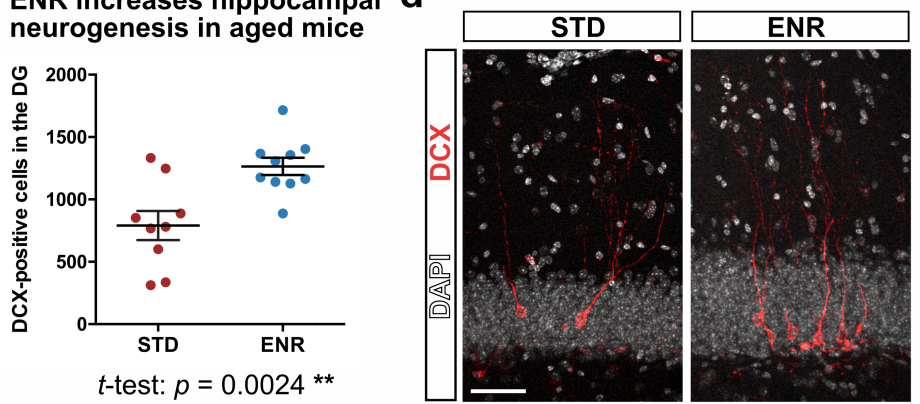

Fig. 4: Functional enrichment connects environmentally sensitive age-related DNA methylation changes with neuronal plasticity and adult hippocampal neurogenesis. a, Integrated map of GO terms, SynGO terms, Reactome pathways and "WikiPathway" enriched among the 676 ENR-induced differentially methylated genes that counteract aging effects. Three main functional clusters were identified (dashed circles), each containing several groups of highly connected terms (yellow circles). Legend indicates node and edge sizes. b, Genes functionally involved in hippocampal neurogenesis are enriched among differentially methylated genes at which ENR counteracts age effects. Hypergeometric test: $p=0.023$. c, Aged mice housed in ENR for one year have significantly more new-born, doublecortin (DCX)-expressing neurons in their dentate gyrus (DG). Depicted are individual sample points with mean and s.e.m. d, Representative image of fluorescent staining to detect new-born neurons in STD and ENR mice. Scale bar: $50 \mu \mathrm{m}$. 


\section{ENR changes DNA methylation in mice at genes associated with age-related cognitive} decline in the human brain

To investigate whether the genes at which ENR counteracted age-related DNA methylation changes in the mouse brain are also dysregulated in the human brain during aging and associated cognitive decline, we overlapped them with three previously published datasets derived from human prefrontal cortex tissue. Dataset 1 contained genes with DNA methylation changes associated with Alzheimer's disease pathology ${ }^{20}$; dataset 2 comprised genes with changes in RNA levels associated with age-related cognitive decline $\mathrm{e}^{36}$ and dataset 3 contained genes with proteomic changes associated with individual cognitive trajectories during aging ${ }^{37}$. Genes of all three datasets methylation changes (Fig. 5a). In total, 256 of the ENR-induced differentially methylated genes detected in the aged mouse brain were also dysregulated in the aged human brain (Fig. 5b; Supplementary Data 7). Among those genes, the most significant enriched biological process from GO analysis was the term "neurogenesis" (adjusted $p=7.9 \mathrm{E}-08 ; 53$ genes) of the higher order group "nervous system development" (adjusted $p=5.0 \mathrm{E}-10 ; 72$ genes). This suggests that the agerelated dysregulation of genes involved in neurogenesis is conserved between mouse and human. The overlap of genes regulated by ENR in mice and genes dysregulated in human brains highlights the intriguing possibility that environmental stimulation could prevent age-related gene dysregulation and associated cognitive decline also in human brains. 
a Enrichment of genes dysregulated in human brain aging and cognitive decline

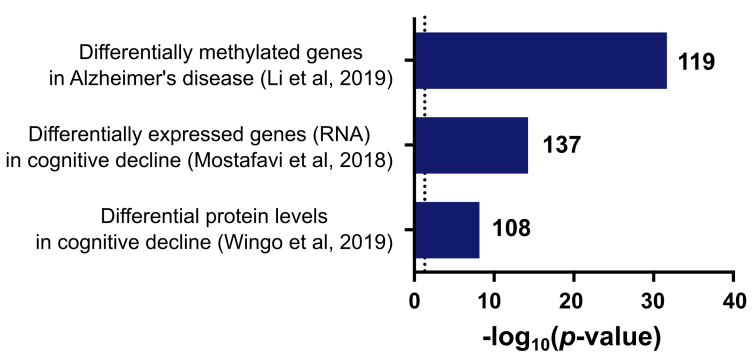

b Network of human brain aging genes counteracted by ENR in mice

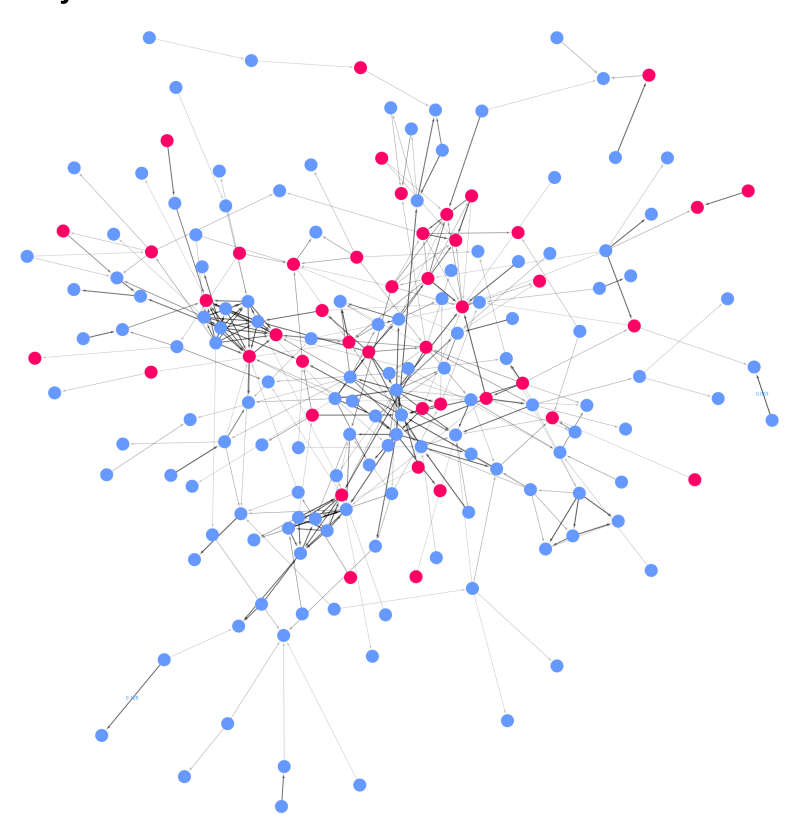

Fig. 5: Genes at which ENR counteracts age-related methylation changes are dysregulated in the human brain in age-related cognitive decline. a, Genes with DNA methylation changes ${ }^{20}$, differential $\mathrm{RNA}^{36}$ or protein ${ }^{37}$ levels in human prefrontal cortex associated with age-related cognitive decline are significantly enriched among the genes that are differentially methylated with aging and ENR in the mouse dentate gyrus. Depicted are the $-\log _{10}$ ( $p$-values) from hypergeometric tests and the number of genes that overlapped between datasets. Dashed line indicates significance threshold of $p=0.05$. b, STRING protein interaction network of human brain aging genes at which ENR counteracted age-related DNA methylation changes in the mouse brain. Genes involved in the highest enriched GO biological process "neurogenesis" are highlighted in red.

\section{Discussion} brain dysfunctions, including reductions in cognition and hippocampal neurogenesis ${ }^{9,10}$. Behavioral interventions and environmental stimulation are known to alter epigenetic 
312 modifications in the brain ${ }^{15,32,39}$, but their influence on age-related epigenetic changes was unclear.

313 Here, we showed that lifelong ENR can counteract age-related DNA methylation changes in the

314 hippocampal dentate gyrus. Many of the genes for which ENR prevented aging-induced differential

315 methylation in the mouse brain were also known to be dysregulated in human brains with aging

316 and related cognitive decline. Our results lend mechanistic support to the potential of behavioral

317 interventions and lifestyle factors to prevent age-related epigenetic changes in the brain in order to

318 improve brain health in old age.

319 We found that ENR prevented the aging-induced loss of CpG methylation genome-wide at binding

320 sites of Mecp2. Since Mecp2 binds methylated cytosines with high affinity broadly across the

321 genome ${ }^{40-42}$, these results suggested that aging is associated with reduced Mecp2 binding to

322 neuronal genomes and that ENR enhances Mecp2 binding in aged brains. Mutations in Mecp2

323 cause Rett syndrome, which is characterized by severe encephalopathy and reduced lifespan ${ }^{43}$.

324 Mecp2 is known to be abundant in neurons and crucial for neuronal development during 325 embryogenesis and in the adult hippocampus ${ }^{44,45}$, but a role of Mecp2 in brain aging had not yet

326 been reported. At the molecular level, Mecp2 has been associated with transcriptional repression, 327 locus-specific gene activation and regulation of alternative splicing ${ }^{41,42,46}$. Therefore, aging328 induced loss of Mecp2 binding as a result of genome-wide CpG hypomethylation might be 329 involved in the aging-associated transcriptional dysregulation and the aberrant splicing of neuronal 330 genes that has been reported for the aged hippocampus ${ }^{47}$. On the other hand, a recent study 331 suggested that Mecp2 is involved in the repression of endogenous repetitive elements in neuronal 332 genomes $^{48}$. ENR-induced methylation of Mecp2 targets could thus prevent the aberrant activation 333 of repetitive elements which is known to contribute to genomic instability in aging ${ }^{49}$. Follow-up 334 studies should experimentally confirm the interaction between CpG hypomethylation and Mecp2 335 binding during brain aging and identify molecular programs downstream of Mecp2 that are 
336 disrupted in the aged brain. Our results suggest not only a possible role of Mecp2 in age-related 337 cognitive decline but also the potential of environmental stimulation to rescue Mecp2 binding in 338 aged brains by DNA methylation of its target sites.

339 With the exception of a few regulated genes ${ }^{50,51}$, the molecular mechanisms underlying ENR340 stimulated brain plasticity were, until now, mostly unknown. Our functional enrichment analyses 341 of differentially methylated genes in the aged mouse dentate gyrus have exposed several pathways 342 potentially underlying the neuroprotective effects of ENR during aging. One such pathway was 343 adult hippocampal neurogenesis and, since increasing neurogenesis promotes aspects of 344 hippocampal function ${ }^{52}$, ENR-stimulated increases in hippocampal neurogenesis likely ameliorate 345 age-related cognitive decline. Further investigation will be needed to determine the cell type 346 specificity of ENR-induced DNA methylation changes in the dentate gyrus and their role in in 347 hippocampal neurogenesis.

348 Since ENR increased adult hippocampal neurogenesis throughout the lifespan, differences in DNA 349 methylation patterns between STD and ENR mice could reflect DNA methylation changes in 350 mature neurons or ENR-induced differences in the cellular composition of the dentate gyrus. Based 351 on our quantifications of new-born neuron numbers, supported by previous quantifications of total 352 granule cell numbers in the dentate gyrus ${ }^{53}$, the percentage of new-born neurons among all neurons 353 increased from $0.21 \%$ in aged STD mice to $0.31 \%$ in aged ENR mice. Because our DNA 354 methylation analysis only considered cytosines with absolute DNA methylation differences larger 355 than $25 \%$ as dmCpGs or dmCpHs, DNA methylation differences as a result of increased 356 percentage of new neurons in ENR mice would have not been detected. Second, because 357 hippocampal neurogenesis sharply decreases in young adulthood and plateaus thereafter ${ }^{54}$, 358 differences in DNA methylation patterns as a result of differences in newborn neuron numbers 359 should have been visible in the 4.5-month-old 'middle-aged' mice. However, the middle-aged mice 
360 showed only minor age-related DNA methylation changes compared to young animals

361 (Supplementary Fig. 4). Therefore, we conclude that the here described ENR-induced DNA

362 methylation changes that counteracted age effects reflect DNA methylation changes in mature

363 hippocampal neurons rather than changes in cellular composition of the dentate gyrus.

364 ENR combines physical and social incentives with continuous novelty and sensory stimulation ${ }^{4}$.

365 Which aspects of ENR and the mechanisms how environmental stimulation prevents age-related

366 DNA methylation changes remains to be investigated. Previous work has shown that exploration

367 of novel environments leads to neuronal activation coupled with short-term and long-lasting

368 changes in transcription and chromatin accessibility in activated hippocampal neurons ${ }^{55,56}$.

369 Additionally, isolated neuronal activation by electroconvulsive stimulation has been shown to lead

370 to widespread changes in DNA methylation, RNA and open chromatin landscapes in the dentate

371 gyrus ${ }^{16,35}$. We showed that ENR-induced differentially methylated genes were significantly

372 enriched for neuronal activity regulated genes, indicating that some of the ENR-induced DNA

373 methylation changes are triggered by neuronal activation. Similarly, Penner et al. reported that age-

374 related DNA methylation changes at the promoter of the neuronal activity-induced gene Egrl were

375 reversed in the hippocampus by acute exploration of a novel environment ${ }^{19}$. We found that many

376 of the genes at which ENR counteracted age-related DNA methylation changes were also

377 differentially methylated by acute (four days) ENR in non-aged brains or by three months ENR in

378 middle-aged mice. Therefore, the rejuvenating effects of ENR on age-related DNA methylation

379 changes are potentially mediated by lifelong, repeated neuronal activation through continuous

380 novelty stimulation in ENR. Future studies should specify how ENR interacts with age-related

381 DNA methylation changes and address whether short-term ENR of aged animals is sufficient to

382 epigenetically rejuvenate the hippocampus. 
383 ENR has an enormous potential to prevent or counteract brain dysfunctions in aged animals, 384 including synaptic plasticity, hippocampal neurogenesis and cognitive abilities ${ }^{57-59}$. Using 385 genome-wide DNA methylation sequencing at single nucleotide resolution, we here demonstrated 386 that ENR also restores a substantial number of age-related DNA methylation changes in the 387 hippocampus. Our data show the rejuvenating effects of ENR at the epigenetic level and provide a 388 potential mechanism how active interaction with the environment supports and promotes brain 389 function throughout the lifespan.

\section{$390 \quad$ Methods}

$391 \quad$ Animals and environmental enrichment

392 Female C57BL/6JRj mice were ordered from Janvier Labs and maintained on a $12 \mathrm{~h}$ light/dark 393 cycle at the animal facility of the Center for Regenerative Therapies Dresden. Food and water were 394 provided ad libitum. At an age of 6 weeks, mice were randomly distributed to an enriched 395 environment or control cages. The enriched environment was a $0.74 \mathrm{~m}^{2}$ enclosure equipped with 396 tunnels and plastic toys. Toys were rearranged once per week, but not in the last four days before 397 analysis. In every experiment, ten mice were housed together in an enriched environment at the 398 same time. Control mice stayed in standard polycarbonate cages (Type II, Tecniplast) in groups of 399 five mice per cage. Dirty bedding material and toys were replaced once per week. The entire 400 enriched environment was cleaned once per month. All experiments were conducted in accordance 401 with the applicable European and national regulations (Tierschutzgesetz) and were approved by 402 the local authority (Landesdirektion Sachsen). 


\section{Reduced Representation Bisulfite Sequencing (RRBS)}

404 Genomic DNA was isolated from micro-dissected dentate gyrus tissue using the QIAamp DNA 405 Micro Kit or the Allprep DNA/RNA Micro Kit (both QIAGEN) following the manufacturer's 406 manuals. RRBS libraries were prepared using the Premium RRBS Kit (Diagenode) and purified 407 twice using Agencourt AMPure XP beads (Beckman Coulter; 1X bead volume). Quality and 408 concentration of RRBS libraries were determined using the High Sensitivity NGS Fragment 409 Analysis Kit (Advanced Analytical) and a fragment analyzer with capillary size of $33 \mathrm{~cm}$ 410 (Advanced Analytical). Sequencing was performed using a HiSeq2500 (experiment with middle411 aged animals; Fig. 1) or a Nextseq (experiment with young and aged animals; Fig. 2-5) platform 412 in a 75 bp single end mode with a minimum sequencing depth of 15 million reads per sample.

$413 \quad$ Bioinformatic data analysis

$414 \quad$ Calculation of differential DNA methylation

415 Fastq reads were trimmed using Trim Galore 0.4.4 and the function Cutadapt 1.8.1. To remove 416 cytosines that were filled in during end preparation, an additional $2 \mathrm{bp}$ were cut off from every 417 sequence with detected adapter. Trimmed reads were mapped against mm10 using 418 Bismark 0.19.0

419 Global DNA methylation levels are the means of the methylation percentages over all cytosines 420 that were covered by RRBS with at least 10 reads in all samples of the respective experiments. 421 Separation into the cytosine contexts $\mathrm{CpG}$ and $\mathrm{CpH}(\mathrm{CHH}$ and $\mathrm{CHG})$ was performed based on $422 \quad$ context annotations extracted from Bismark.

423 Detection of differentially methylated cytosines was performed using methylKit $1.5 .2^{61}$. Briefly, 424 methylation levels were extracted from sorted Binary Alignment Map files using the function 425 processBismarkAln. Data was filtered for CpGs with a minimum coverage of ten reads and a 
maximum coverage of $99.9 \%$ percentile using the function filterByCoverage. Using the function unite, CpGs were selected that were sufficiently covered in at least three samples per group. Differentially methylated cytosines were identified using the methDiff function applying the chisquared test, a significance threshold of $q<0.001$ and a threshold for absolute DNA methylation differences higher than $25 \%$.

431 Differentially methylated cytosines were annotated to the gene with the nearest transcription start 432 site using data tables downloaded from Ensembl BioMart (as of 01.05.2019) ${ }^{62}$. Genes with at least 433 four annotated differentially methylated cytosines were considered differentially methylated genes. 434 Gene names used in this study are Ensembl gene names. Corresponding Entrez identifiers were 435 retrieved from the Bioconductor package AnnotationDbi ${ }^{63}$ using Ensembl gene identifiers as keys. 436 Mapping of mouse genes to homologous human genes was performed using data tables 437 downloaded from Ensembl BioMart ${ }^{62}$.

\section{$438 \quad$ Genomic feature annotation}

439 Genomic coordinates of $\mathrm{CpG}$ islands, exons and introns were downloaded from the UCSC genome 440 browser ${ }^{64}$. CpG island shores were defined as $2 \mathrm{~kb}$ upstream and downstream of a CpG island. 441 Promoter regions were determined as $\pm 1 \mathrm{~kb}$ from the transcription start sites of all known 442 transcripts. Enhancer and transcription factor binding sites were downloaded from the Ensembl 443 Regulatory Build track of the UCSC genome browser ${ }^{65}$. Superenhancer regions are from adult 444 mouse cortex and were retrieved from dbSUPER ${ }^{66}$. Overlaps of cytosines with genomic regions or 445 other cytosines were performed using the functions subsetByOverlaps or findOverlaps of the $\mathrm{R}$ 446 package Genomic Ranges $3.7^{67}$. Genomic feature enrichment was analyzed using count tables of 447 feature coverage of differentially methylated cytosines and all cytosines covered by RRBS 448 (background). Odds ratios and $p$-values of feature enrichment were calculated by fitting a general 
449 linear model with binomial distribution using the function $g l m$ in R. Odds ratios are the exponential $450 \quad$ coefficients of the model.

$451 \quad$ Transcription factor binding analysis

452 The number of differentially methylated cytosines that overlap with position weight matrices of 453 transcription factor binding motifs was determined using the Biostrings package with a minimum 454 match score of $90 \%$. Transcription factor motifs were retrieved from motifDb ${ }^{68}$. Motif enrichment 455 for each transcription factor was tested by applying hypergeometric tests using the function $456 \operatorname{phyper}(q, m, n, k)+\operatorname{dhyper}(q, m, n, k)$ with $\mathrm{q}=$ number of differentially methylated cytosines 457 overlapping with the motif, $\mathrm{m}=$ number of background cytosines overlapping with the motif, $\mathrm{n}=$ 458 number of background cytosines not overlapping with the motif and $\mathrm{k}=$ total number of 459 differentially methylated cytosines. Multiple testing correction of $p$-values was performed using 460 the FDR method.

461 For conformation of Mecp2 binding sites, Mecp2 ChIP-Seq data were downloaded from GEO 462 (GSE67293) and overlapped with dmCpGs using Genomic Ranges 3.7. Hypergeometric test for 463 the enrichment analysis was performed as described for the motif analysis.

$464 \quad$ Functional gene enrichment analyses

465 Enrichment for gene ontology (GO) cellular components was analyzed using the online tool 466 GREAT $^{69}$. Pathway analysis was performed using the R package ReactomePA ${ }^{70}$ with a minimum 467 of 5 and a maximum of 300 genes per pathway. Enrichment for genes involved in synaptic 468 processes was performed using the SynGO online tool (https://www.syngoportal.org/) with default 469 settings. All enrichment analyses were performed with differentially methylated genes as query $470 \quad$ lists and all genes covered by RRBS as background lists.

471 Gene set enrichment was analyzed by performing hypergeometric tests using the function $472 \operatorname{phyper}(q, m, n, k)+\operatorname{dhyper}(q, m, n, k)$ in $\mathrm{R}$ with $\mathrm{q}=$ number of differentially methylated genes 
473 overlapping with the gene set, $\mathrm{m}=$ number of background genes overlapping with the gene set, $\mathrm{n}=$

474 number of background genes not overlapping with the gene set and $\mathrm{k}=$ total number of 475 differentially methylated genes. Genes involved in adult hippocampal neurogenesis were 476 downloaded from the Mammalian Adult Neurogenesis Gene Ontology (MANGO) v3.2 ${ }^{14}$. The gene 477 list was filtered for genes with reported positive or negative effect on neurogenesis after protein 478 manipulation or gene manipulation (in total 283 genes). Genes or cytosines with known aging479 induced DNA methylation changes were extracted from ${ }^{38,71,72}$ for peripheral tissues and from ${ }^{18,28}$ 480 for the hippocampus.

481 The enrichment map was generated according to Merico et al. ${ }^{33}$ using genes that contain at least 482 four differentially methylated cytosines (dmCpGs or dmCpHs). GO biological process, GO cellular 483 component, Reactome, and Wikipathways enrichment analyses were performed in g:Profiler 484 (https://biit.cs.ut.ee/gprofiler) using gene ensembl identifiers. The results from SynGO and 485 MANGO enrichment analysis were integrated into the gmt file downloaded from g:Profiler. The 486 network was generated using the app Enrichment Map 3.2.0 in cytoscape 3.7.1 using a $q$-value 487 threshold (FDR) of 0.05 . Clusters were manually annotated. Groups with fewer than six connected 488 terms were not drawn. The STRING protein interaction network was generated inside cyotscape 489 3.7.1 using human ensembl identifiers with the plugin STRING (protein query) at a confidence $490 \quad$ score cutoff of 0.5 with automated enrichment analysis.

$491 \quad$ Data and code availability

492 Sequencing data are deposited at GEO (accession number pending). Sequencing data were 493 analyzed using in-house R scripts which are available upon request.

$494 \quad$ Analysis of adult hippocampal neurogenesis

495 Tissue fixation and immunofluorescent stainings for the detection of new-born neurons were 496 performed as previously described ${ }^{34}$. Briefly, mice were anesthetized with $100 \mathrm{mg} / \mathrm{kg} \mathrm{ketamine}$ 
497 (WDT) and $10 \mathrm{mg} / \mathrm{kg}$ xylazin (Serumwerk Bernburg AG) and transcardially perfused with $0.9 \%$ 498 sodium chloride. Brains were removed from the skull and one hemisphere fixed in $4 \%$ 499 paraformaldehyde prepared in phosphate buffer ( $\mathrm{pH} 7.4)$ overnight at $4^{\circ} \mathrm{C}$. Brains were incubated $500 \quad$ in $30 \%$ sucrose in phosphate buffer for two days and cut into $40 \mu \mathrm{m}$ coronal sections using a dry501 ice-cooled copper block on a sliding microtome (Leica, SM2000R). Sections were stored at $4{ }^{\circ} \mathrm{C}$ 502 in cryoprotectant solution (25\% ethyleneglycol, $25 \%$ glycerol in $0.1 \mathrm{M}$ phosphate buffer, $\mathrm{pH} 7.4$ ). 503 For immunofluorescent stainings, sections were washed and unspecific binding sites were blocked 504 in phosphate buffered saline supplemented with $10 \%$ donkey serum (Jackson Immuno Research 505 Labs) and $0.2 \%$ Triton X-100 (Carl Roth) for $1 \mathrm{~h}$ at room temperature. Primary antibodies were 506 applied overnight at $4{ }^{\circ} \mathrm{C}$ as follows: polyclonal goat anti-doublecortin (1:250; Santa Cruz), 507 polyclonal rat anit-Ki67 (1:500; eBioscience), polyclonal goat anti-Sox2 (1:500; Santa Cruz). The 508 secondary antibodies anti-goat Cy3 and anti-rat 488 (1:1000; Jackson Immuno Research Labs) 509 were incubated for $2 \mathrm{~h}$ at room temperature. Antibodies were diluted in phosphate buffered saline 510 supplemented with $3 \%$ donkey serum and $0.2 \%$ Triton X-100. Nuclei were labeled with 4',6511 diamidino-2-phenylindole (DAPI; 1:4000; Jackson Immuno Research Labs). Sections were 512 mounted onto glass slides and cover-slipped using Aquamount (Polysciences Inc.). Stainings were 513 imaged using a Zeiss Apotome equipped with an AxioCam MRm camera and the software 514 AxioVision 4.8 (Zeiss). Cells were quantified in every sixth section along the rostro-caudal axis of 515 the dentate gyrus.

\section{$516 \quad$ Statistics}

517 The statistical tests used are reported in the specific results or methods section. All $t$-tests were 518 two-sided and performed using GraphPad Prism 6.0. When performing multiple comparisons, $p$ 519 values were adjusted using FDR corrections. All measurements were taken from distinct samples. 


\section{Acknowledgments}

521 This study was financed from basic institutional funds. S.Z. was supported by an EMBO Short522 Term Fellowship and a fellowship from the International Max Planck Research School on the Life 523 Course, Berlin. The authors thank Hongjun Song for discussion and methodological support.

\section{$524 \quad$ Author contributions}

525 Conceptualization - S.Z., G.K.; Investigation/ Visualization - S.Z.; Methodology/ Formal analysis 526 - S.Z., R.W.O; Software/ Data Curation - R.W.O., M.L., S.Z.; Resources - A.D., G.K.; Writing 527 original draft - S.Z.; Writing review and editing - S.Z., R.W.O., G.K..

$528 \quad$ Competing interests

$529 \quad$ None declared. 


\section{References}

1. Phillips, C. Lifestyle Modulators of Neuroplasticity: How Physical Activity, Mental Engagement, and Diet Promote Cognitive Health during Aging. Neural Plast. (2017).

2. Cabeza, R. et al. Maintenance, reserve and compensation: the cognitive neuroscience of healthy ageing. Nat. Rev. Neurosci. 19, 701-710 (2018).

3. Stern, Y. et al. Whitepaper: Defining and investigating cognitive reserve, brain reserve, and brain maintenance. Alzheimer's Dement. 1-7 (2018).

4. Kempermann, G. Environmental enrichment, new neurons and the neurobiology of individuality. Nat. Rev. Neurosci. (2019).

5. Rogers, J., Renoir, T. \& Hannan, A. J. Gene-environment interactions informing therapeutic approaches to cognitive and affective disorders. Neuropharmacology 145, 3748 (2019).

6. Fan, X., Wheatley, E. G. \& Villeda, S. A. Mechanisms of Hippocampal Aging and the Potential for Rejuvenation. Annu. Rev. Neurosci. 40, 251-272 (2017).

7. Blasco, M. A., Partridge, L., Serrano, M., Kroemer, G. \& Lo, C. The Hallmarks of Aging. Cell 153, 1194-1217 (2013).

8. Horvath, S. DNA methylation age of human tissues and cell types. Genome Biol. 14, R115 (2013).

9. Oliveira, A. M. M., Hemstedt, T. J. \& Bading, H. Rescue of aging-associated decline in Dnmt3a2 expression restores cognitive abilities. Nat. Neurosci. 15, 1111-1113 (2012).

10. Gontier, G. et al. Tet2 Rescues Age-Related Regenerative Decline and Enhances Cognitive Function in the Adult Mouse Brain. Cell Rep. 22, 2094-2106 (2018).

11. Kaas, G. A. et al. TET1 Controls CNS 5-Methylcytosine Hydroxylation, Active DNA Demethylation, Gene Transcription, and Memory Formation. Neuron 79, 1086-1093 (2013).

12. Feng, J. et al. Dnmt1 and Dnmt3a maintain DNA methylation and regulate synaptic function in adult forebrain neurons. Nat. Neurosci. 13, 423-430 (2010).

13. Nelson, E. D., Kavalali, E. T. \& Monteggia, L. M. Activity-Dependent Suppression of Miniature Neurotransmission through the Regulation of DNA Methylation. J. Neurosci. 28, 395-406 (2008).

14. Weaver, I. C. G. et al. Epigenetic programming by maternal behavior. Nat. Neurosci. 7, 847-54 (2004).

15. Halder, R. et al. DNA methylation changes in plasticity genes accompany the formation and maintenance of memory. Nat. Neurosci. 19, 102-110 (2016).

16. Guo, J. U. et al. Neuronal activity modifies the DNA methylation landscape in the adult brain. Nat. Neurosci. 14, 1345-1351 (2011).

17. Cholewa-Waclaw, J. et al. The Role of Epigenetic Mechanisms in the Regulation of Gene Expression in the Nervous System. J. Neurosci. 36, 11427-11434 (2016).

18. Masser, D. R. et al. Sexually divergent DNA methylation patterns with hippocampal 
aging. Aging Cell 16, 1342-1352 (2017).

19. Penner, M. R. et al. Age-related changes in Egr1 transcription and DNA methylation within the hippocampus. Hippocampus 26, 1008-20 (2016).

20. Li, P. et al. Epigenetic dysregulation of enhancers in neurons is associated with Alzheimer's disease pathology and cognitive symptoms. Nat. Commun. 10, 2246 (2019).

21. Gasparoni, G. et al. DNA methylation analysis on purified neurons and glia dissects age and Alzheimer's disease-specific changes in the human cortex. Epigenetics Chromatin 11, 41 (2018).

22. Meissner, A. et al. Reduced representation bisulfite sequencing for comparative highresolution DNA methylation analysis. Nucleic Acids Res. 33, 5868-5877 (2005).

23. Boyle, P. et al. Gel-free multiplexed reduced representation bisulfite sequencing for largescale DNA methylation profiling. Genome Biol. 13, R92 (2012).

24. The Gene Ontology Resource: 20 years and still GOing strong. Nucleic Acids Res. 47, D330-D338 (2019).

25. Fabregat, A. et al. The Reactome Pathway Knowledgebase. Nucleic Acid Res. 46, 649-655 (2018).

26. Overall, R. W., Paszkowski-Rogacz, M. \& Kempermann, G. The Mammalian Adult Neurogenesis Gene Ontology (MANGO) Provides a Structural Framework for Published Information on Genes Regulating Adult Hippocampal Neurogenesis. PLoS One 7, (2012).

27. Koopmans, F. et al. SynGO: An Evidence-Based, Expert-Curated Knowledge Base for the Synapse. Neuron 103, 217-234 (2019).

28. Hadad, N. et al. Caloric restriction mitigates age-associated hippocampal differential CG and non-CG methylation. Neurobiol. Aging 67, 53-66 (2018).

29. Rinaldi, L. et al. Dnmt3a and Dnmt3b Associate with Enhancers to Regulate Human Epidermal Stem Cell Homeostasis. Cell Stem Cell 19, 491-501 (2016).

30. Nan, X., Campoy, F. J. \& Bird, A. MeCP2 Is a Transcriptional Repressor with Abundant Binding Sites in Genomic Chromatin. Cell 88, 471-481 (1997).

31. Gabel, H. W. et al. Disruption of DNA-methylation-dependent long gene repression in Rett syndrome. Nature 522, 89-93 (2015).

32. Stroud, H. et al. Early-Life Gene Expression in Neurons Modulates Lasting Epigenetic States. Cell 171, 1151-1154 (2017).

33. Merico, D., Isserlin, R., Stueker, O., Emili, A. \& Bader, G. D. Enrichment map: A network-based method for gene-set enrichment visualization and interpretation. PLoS One 5, (2010).

34. Kempermann, G., Kuhn, H. G. \& Gage, F. H. Experience-induced neurogenesis in the senescent dentate gyrus. J. Neurosci. 18, 3206-3212 (1998).

35. Su, Y. et al. Neuronal activity modifies the chromatin accessibility landscape in the adult brain. Nat. Neurosci. 20, 476-483 (2017).

36. Mostafavi, S. et al. A molecular network of the aging human brain provides insights into 
608

609

610

611

612

613

614

615

616

617

618

619

620

621

622

623

624

625

626

627

628

629

630

631

632

633

634

635

636

637

638

639

640

641

642

643

644

645

646

the pathology and cognitive decline of Alzheimer's disease. Nat. Neurosci. 21, 811-819 (2018).

37. Wingo, A. P. et al. Large-scale proteomic analysis of human brain identifies proteins associated with cognitive trajectory in advanced age. Nat. Commun. 10, 1619 (2019).

38. Maegawa, S. et al. Caloric restriction delays age-related methylation drift. Nat. Commun. 8, (2017).

39. Zhang, T. Y. et al. Environmental enrichment increases transcriptional and epigenetic differentiation between mouse dorsal and ventral dentate gyrus. Nat. Commun. 9, 1-11 (2018).

40. Lewis, J. D. et al. Purification, sequence, and cellular localization of a novel chromosomal protein that binds to Methylated DNA. Cell 69, 905-914 (1992).

41. Gabel, H. W. et al. Disruption of DNA-methylation-dependent long gene repression in Rett syndrome. Nature (2015). doi:10.1038/nature14319

42. Kinde, B., Wu, D. Y., Greenberg, M. E. \& Gabel, H. W. DNA methylation in the gene body influences MeCP2-mediated gene repression. Proc. Natl. Acad. Sci. U. S. A. 113, 15114-15119 (2016).

43. Guy, J., Cheval, H., Selfridge, J. \& Bird, A. The Role of MeCP2 in the Brain. Annu. Rev. Cell Dev. Biol. 27, 631-652 (2011).

44. Ip, J. P. K., Mellios, N. \& Sur, M. Rett syndrome: insights into genetic, molecular and circuit mechanisms. Nat. Rev. Neurosci. 19, 368-382 (2018).

45. Li, H. et al. Cell cycle-linked MeCP2 phosphorylation modulates adult neurogenesis involving the Notch signalling pathway. Nat. Commun. 5, 5601 (2014).

46. Osenberg, S. et al. Activity-dependent aberrations in gene expression and alternative splicing in a mouse model of Rett syndrome. Proc. Natl. Acad. Sci. U. S. A. 115, E5363E5372 (2018).

47. Stilling, R. M. et al. De-regulation of gene expression and alternative splicing affects distinct cellular pathways in the aging hippocampus. Front. Cell. Neurosci. 1-15 (2014). doi:10.3389/fncel.2014.00373

48. Zhao, B. et al. Somatic LINE-1 retrotransposition in cortical neurons and non-brain tissues of Rett patients and healthy individuals. PLOS Genet. 15, e1008043 (2019).

49. Cardelli, M. The epigenetic alterations of endogenous retroelements in aging. Mech. Ageing Dev. 174, 30-46 (2018).

50. Rampon, C. et al. Effects of environmental enrichment on gene expression in the brain. PNAS 97, 12880-12884 (2000).

51. Zhang, Y. et al. Transcriptomics of Environmental Enrichment Reveals a Role for Retinoic Acid Signaling in Addiction. Front. Mol. Neurosci. 9, (2016).

52. Sahay, A. et al. Increasing adult hippocampal neurogenesis is sufficient to improve pattern separation. Nature 472, 466-470 (2011). 
53. Kempermann, G. \& Gage, F. H. Experience-Dependent Regulation of Adult Hippocampal Neurogenesis: Effects of Long-Term Stimulation and Stimulus Withdrawal. Hippocampus 9, 321-332 (1999).

54. Ben Abdallah, N. M. B., Slomianka, L., Vyssotski, A. L. \& Lipp, H. P. Early age-related changes in adult hippocampal neurogenesis in C57 mice. Neurobiol. Aging 31, 151-161 (2010).

55. Jaeger, B. N. et al. A novel environment-evoked transcriptional signature predicts reactivity in single dentate granuel neurons. Nat. Commun. 9, 3084 (2018).

56. Fernandez-Albert, J. et al. Immediate and deferred epigenomic signatures of in vivo neuronal activation in mouse hippocampus. Nat. Neurosci. 1-13 (2019). doi:10.1038/s41593-019-0476-2

57. Kempermann, G., Gast, D. \& Gage, F. H. Neuroplasticity in Old Age: Sustained Fivefold Induction of Hippocampal Neurogenesis by Long-term Environmental Enrichment. Ann. Neurol. 52, 135-143 (2002).

58. Cortese, G. P., Olin, A., O’Riordan, K., Hullinger, R. \& Burger, C. Environmental enrichment improves hippocampal function in aged rats by enhancing learning and memory, LTP, and mGluR5-Homer1c activity. Neurobiol. Aging 63, 1-11 (2018).

59. Speisman, R. B. et al. Environmental enrichment restores neurogenesis and rapid acquisition in aged rats. Neurobiol. Aging 34, 263-274 (2013).

60. Krueger, F. \& Andrews, S. R. Bismark : a flexible aligner and methylation caller for Bisulfite-Seq applications. Bioinformatics 27, 1571-1572 (2011).

61. Akalin, A. et al. methylKit: a comprehensive R package for the analysis of genome-wide DNA methylation profiles. Genome Biol. 13, R87 (2012).

62. Kinsella, R. J. et al. Ensembl BioMarts : a hub for data retrieval across taxonomic space. Database 2011:bar03, (2011).

63. Pagès, H., Carlson, M., Falcon, S. \& Li, N. AnnotationDbi: Annotation Database Interface. R package version 1.42.1. (2018).

64. Karolchik, D. et al. The UCSC Table Browser data retrieval tool. Nucleic Acids Res. 32, 493-496 (2004).

65. Zerbino, D. R., Wilder, S. P., Johnson, N., Juettemann, T. \& Flicek, P. R. The Ensembl Regulatory Build. Genome Biol. 16, 1-8 (2015).

66. Khan, A. \& Zhang, X. dbSUPER: a database of super-enhancers in mouse and human genome. Nucleic Acids Res. 44, D164-71 (2016).

67. Lawrence, M. et al. Software for Computing and Annotating Genomic Ranges. PLOS Comput. Biol. 9, 1-10 (2013).

68. Shannon, P. \& Richards, M. MotifDb: An Annotated Collection of Protein-DNA Binding Sequence Motifs. R Packag. version 1.26.0. (2019).

69. Mclean, C. Y. et al. GREAT improves functional interpretation of cis -regulatory regions. Nat. Biotechnol. 28, 495-501 (2010). 
70. Yu, G. \& He, Q. Molecular BioSystems ReactomePA : an R / Bioconductor package for reactome pathway analysis and visualization. Mol. Biosyst. 12, 477-479 (2016).

71. Stubbs, T. M. et al. Multi-tissue DNA methylation age predictor in mouse. Genome Biol. 18, (2017).

72. Thompson, M. J. et al. A multi-tissue full lifespan epigenetic clock for mice. Aging (Albany. NY). 10, 2832-2854 (2018). 


\section{Epigenetic rejuvenation of the hippocampus by environmental enrichment}

Sara Zocher ${ }^{1,2}$, Rupert W. Overall ${ }^{1,2}$, Mathias Lesche ${ }^{2,3}$, Andreas Dahl' ${ }^{2,3}$, Gerd

Kempermann ${ }^{1,2^{*}}$

${ }^{1}$ German Center for Neurodegenerative Diseases (DZNE) Dresden, Tatzberg 41, 01307

Dresden, Germany

${ }^{2}$ Center for Regenerative Therapies Dresden (CRTD), Technische Universität Dresden,

Fetscherstraße 105, 01307 Dresden, Germany

${ }^{3}$ DRESDEN-concept Genome Center c/o Center for Molecular and Cellular Bioengineering

(CMCB), Technische Universität Dresden, Fetscherstraße 105, 01307 Dresden, Germany

*Corresponding author. E-mail: Gerd.Kempermann@dzne.de

\section{Content:}

Supplementary Figures 1-6 with figure legends

Description of Supplementary Data files 1-7 
bioRxiv preprint doi: https://doi.org/10.1101/776310; this version posted September 23, 2019. The copyright holder for this preprint (which was not certified by peer review) is the author/funder, who has granted bioRxiv a license to display the preprint in perpetuity. It is made available

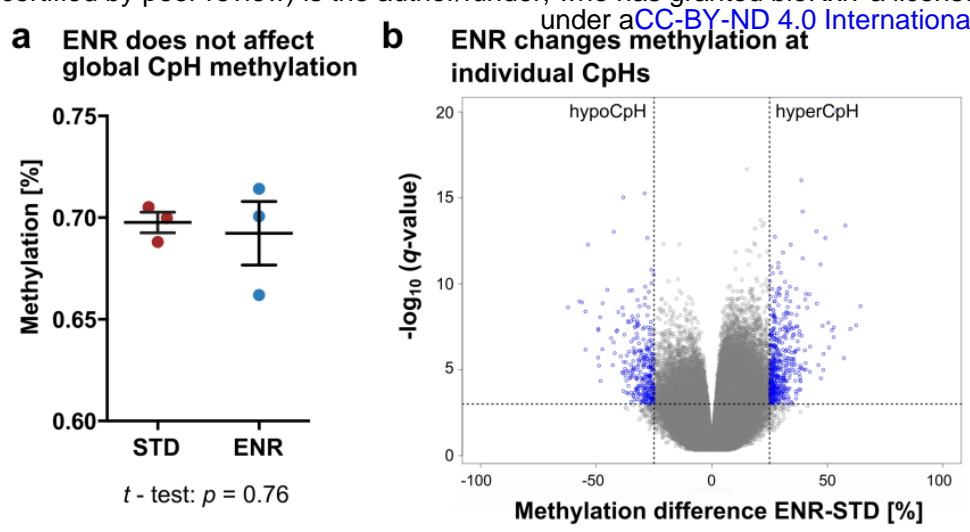

C Genomic feature annotation of $\mathrm{dmCpHs}$ global $\mathrm{CpH}$ methylation

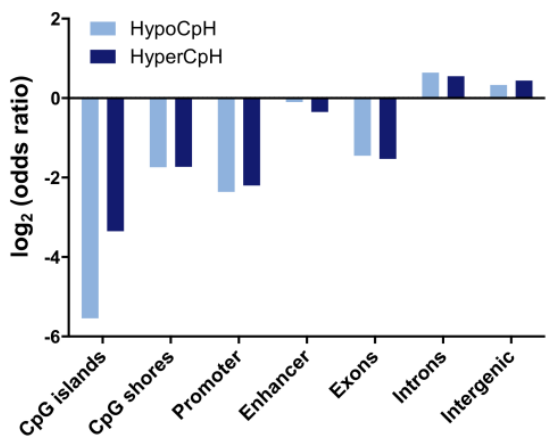

\section{Supplementary Fig. 1}

Environmental enrichment elicits locus-specific $\mathrm{CpH}$ methylation changes in the adult, non-aged dentate gyrus. DNA methylation profiles from 4.5 months old mice housed in ENR or STD for three months. a, ENR does not change global $\mathrm{CpH}$ methylation. Depicted are individual data points with mean and s.e.m. b, Volcano plot depicting differentially methylated $\mathrm{CpHs}(\mathrm{dmCpHs})$ in blue. In total, $750 \mathrm{dmCpHs}$ were detected $(0.019 \%$ of covered $\mathrm{CpHs})$. Of those, $38.93 \%$ were hypomethylated (hypoCpH) and $61.07 \%$ were hypermethylated (hyperCpH) in ENR mice. c, Genomic distribution of hypoCpH and hyperCpH (adjusted $p<0.001$ for $\mathrm{CpG}$ islands, $\mathrm{CpG}$ island shores, promoters, exons, introns; adjusted $p<0.05$ for intergenic regions; enhancer: adjusted $p=0.65$ for hypoCpH, adjusted $p=0.26$ for hyperCpH). 
bioRxiv preprint doi: https://doi.org/10.1101/776310; this version posted September 23, 2019. The copyright holder for this preprint (which was not certified by peer review) is the author/funder, who has granted bioRxiv a license to display the preprint in perpetuity. It is made available

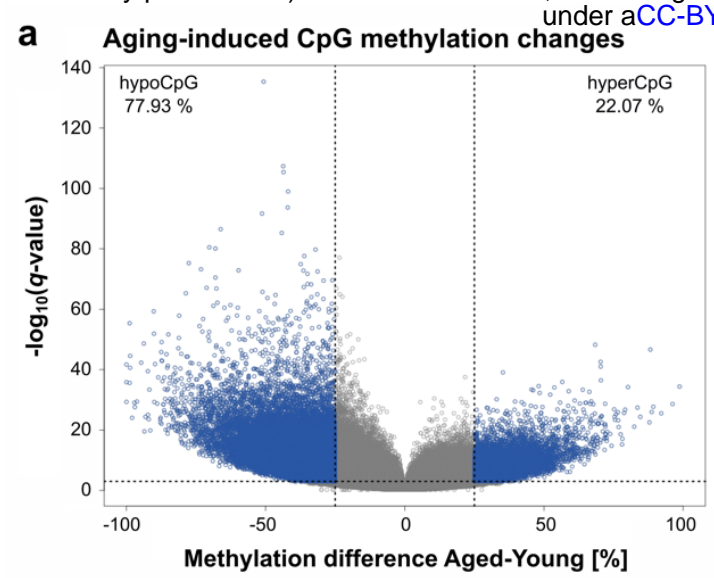

International license.

b Aging-induced $\mathrm{CpH}$ methylation changes

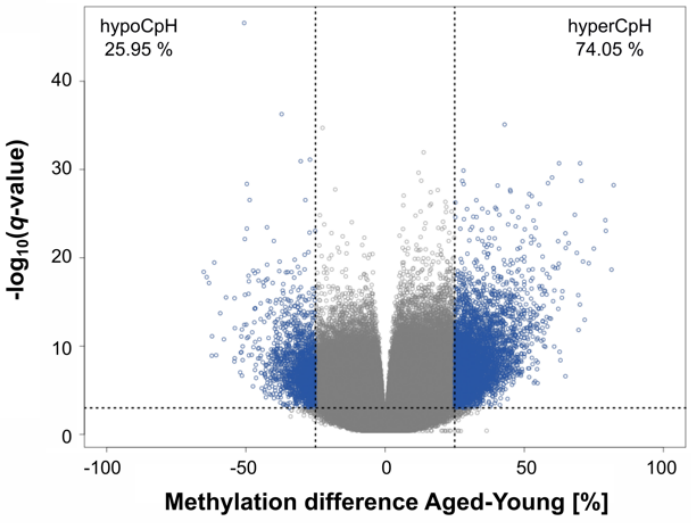
genes with published datasets

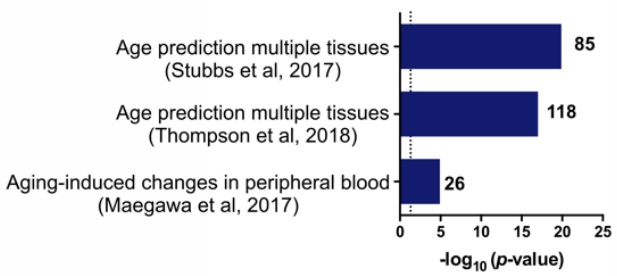

d Shared enriched pathways between aging-induced and ENR-induced methylation changes Enriched pathways Aging ENR

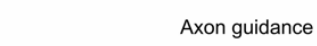

NRAGE signals death through JNK G alpha (12/13) signalling events Neuronal System Rho GTPase cycle Protein-protein interactions at synapses Extracellular matrix organization Signalling by NGF Platelet activation, signaling and aggregation Platelet Aggregation (Plug Formation) RET signaling

Trafficking of AMPA receptors Glutamate binding, activation of AMPA receptors and synaptic plasticity Signaling by SCF-KIT p75 NTR receptor-mediated signalling Nuclear Receptor transcription pathway Integration of energy metabolism Cell death signalling via NRAGE, NRIF and NADE

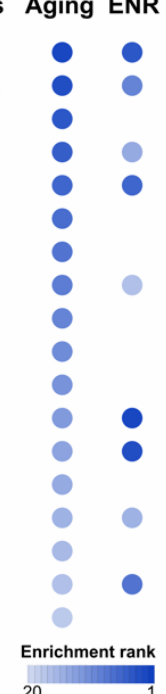

\section{Supplementary Fig. 2}

Age-related $\mathrm{CpG}$ and $\mathrm{CpH}$ methylation changes in the dentate gyrus. a, Volcano plot depicting CpGs where aging changed methylation in STD mice in blue ( $\mathrm{n}=5$ for young mice; $\mathrm{n}=7$ for aged mice). In total, $5.51 \%$ of all covered $\mathrm{CpGs}$ were dmCpGs. Age-related $\mathrm{CpG}$ hypomethylation ( $77.93 \%$ of dmCpGs) is more prominent than $\mathrm{CpG}$ hypermethylation (22.07\% of dmCpGs). b, Aging changed methylation at $0.17 \%$ of $\mathrm{CpHs}$ in the genome. The vast majority of $\mathrm{dmCpHs}$ were hypermethylated in the aged dentate gyrus $(74.05 \%)$. c, Enrichment of age-related differentially methylated genes ( $\mathrm{CpG}$ and $\mathrm{CpH}$ context) from published dataset among genes with age-related $\mathrm{CpG}$ or $\mathrm{CpH}$ methylation changes in the dentate gyrus ( $p$-values from hypergeometric tests). d, Top 18 pathways from Reactome pathway analysis with age-related differentially methylated genes (in total 3983 genes). Pathways were ordered by significance of the enrichment and colored based on the rank of significance. Among the top 18 enriched pathways from age-related methylation changes are also the highest enriched pathways from ENR-induced DNA methylation changes in non-aged mice after three months of ENR. 
bioRxiv preprint doi: https://doi.org/10.1101/776310; this version posted September 23, 2019. The copyright holder for this preprint (which was not certified by peer review) is the author/funder, who has granted bioRxiv a license to display the preprint in perpetuity. It is made available No global $\mathrm{CpH}$ methylation changes under aCC-BY-ND 4.0 International license.

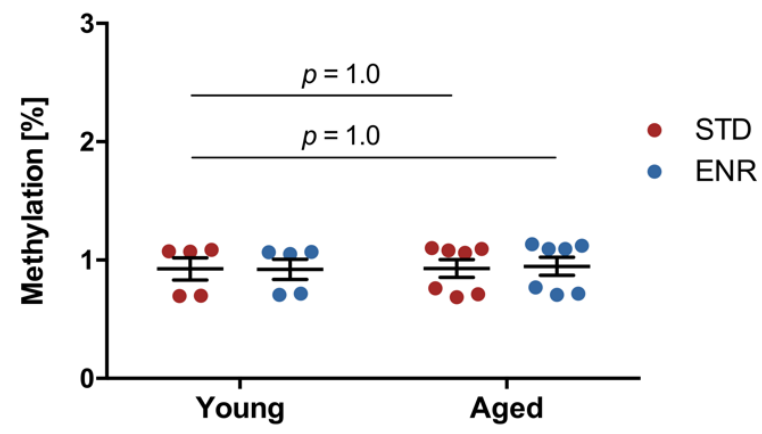

\section{Supplementary Fig. 3}

No interaction between environmental enrichment and aging in global $\mathrm{CpH}$ methylation. Aging and ENR do not change global $\mathrm{CpH}$ methylation (mean over 246,878 CpHs). The $p$-values are from two-way ANOVA with Dunnett's post hoc test. 
bioRxiv preprint doi: https://doi.org/10.1101/776310; this version posted September 23, 2019. The copyright holder for this preprint (which was not certified by peer review) is the author/funder, who has granted bioRxiv a license to display the preprint in perpetuity. It is made available

a $\mathrm{CpG}$ and $\mathrm{CpH}$ methylation levels of middle-aged (4.5 months) mice are similar to young animals
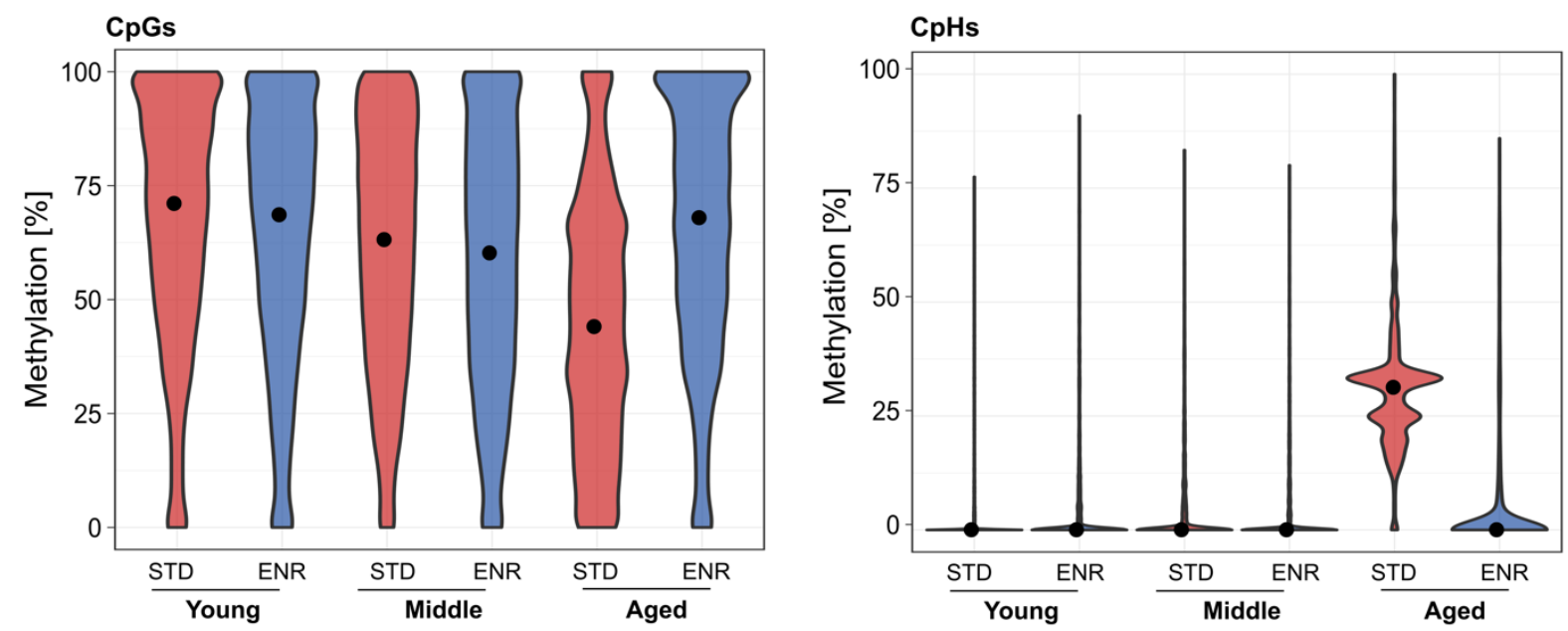

b ENR counteracts age-realted $\mathrm{CpG}$ and $\mathrm{CpH}$ methylation changes that occur from middle-aged to aged animals

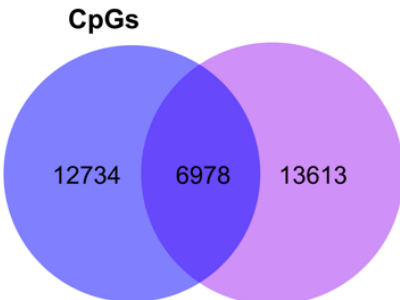

Aging

hypoCpG
ENR 1․

hyperCpG

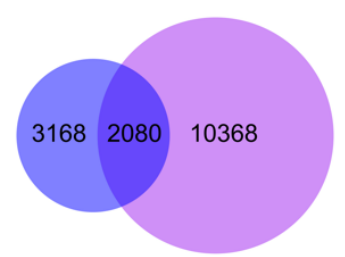

Aging 1 hyperCpG

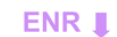

hypoCpG

$\mathrm{CpHs}$

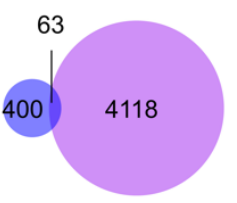

Aging hypoCpH hyperCpH

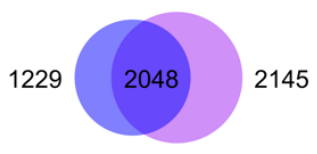

Aging 1 hyperCpH
ENR V. hypoCpH

\section{Supplementary Fig. 4}

The interaction of age-related and ENR-induced DNA methylation changes between middleaged and aged animals. a, STD and ENR housed middle-aged animals (4.5 months) show CpG and $\mathrm{CpH}$ methylation levels similar to young animals. b, In total, $36.29 \%$ of $\mathrm{CpG}$ methylation differences between 4.5-month-old and 14-month-old STD mice and 56.44\% of $\mathrm{CpH}$ methylation changes were counteracted by ENR. 
bioRxiv preprint doi: https://doi.org/10.1101/776310; this version posted September 23, 2019. The copyright holder for this preprint (which was not certified by peer review) is the author/funder, who has granted bioRxiv a license to display the preprint in perpetuity. It is made available

a Precursor proliferation b under aCC-BY-ND 4.0 International license.
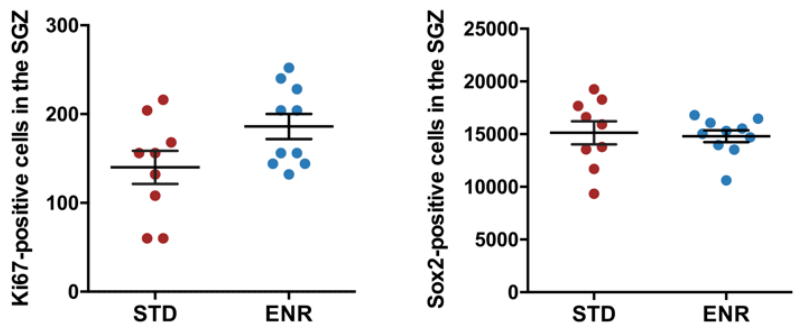

t-test: $p=0.063$

$t$-test: $p=0.79$

\section{Supplementary Fig. 5}

Influence of life-long ENR on neuronal precursor cells in the dentate gyrus. a, Quantification of the numbers of proliferating, Ki67-positive precursor cells in the subgranular zone (SGZ). b, No difference in total numbers of Sox2-positive precursor cells in the SGZ between aged mice housed in STD or ENR for one year. 
bioRxiv preprint doi: https://doi.org/10.1101/776310; this version posted September 23, 2019. The copyright holder for this preprint (which was not certified by peer review) is the author/funder, who has granted bioRxiv a license to display the preprint in perpetuity. It is made available

a Overlap of ENR-induced differentially thethypaftedgeñell 4.0 International license. at different ages

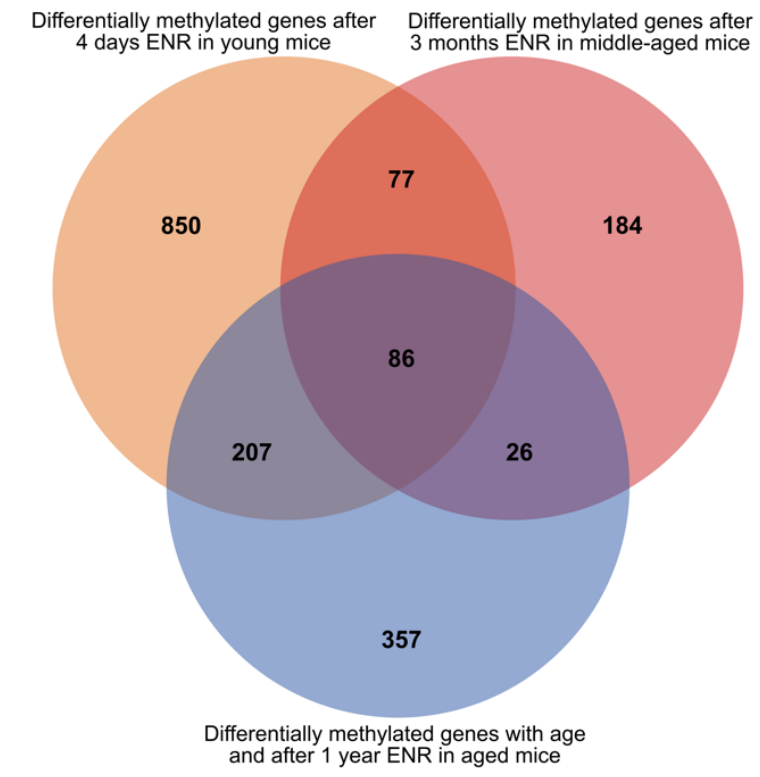

b Enrichment of genes regulated by neuronal activity

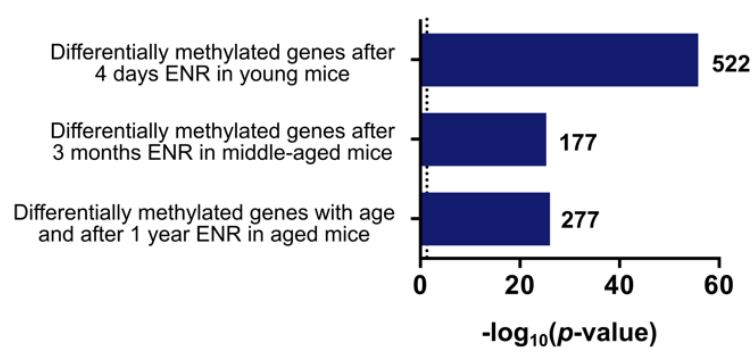

\section{Supplementary Fig. 6}

Genes at which ENR counteracts age-related DNA methylation changes overlap with ENRinduced methylation changes in the non-aged brain and with neuronal activity regulated genes. a, Venn diagram with number of overlapping genes between groups. b, Enrichment of genes that change RNA levels after activation of neurons in the dentate gyrus by electroconvulsive stimulation. Depicted are the $-\log _{10}(p$-values) and the number of overlapping genes for every dataset. 
bioRxiv preprint doi: https://doi.org/10.1101/776310; this version posted September 23, 2019. The copyright holder for this preprint (which was not certified by peer review) is the author/funder, who has granted bioRxiv a license to display the preprint in perpetuity. It is made available

Captions for Supplementary Dahar fif $\bar{E}^{-B Y-N D} 4.0$ International license.

\section{Supplementary Data 1}

Differentially methylated cytosines (sheet 1) and differentially methylated genes (sheet 2) in the dentate gyrus of 4.5-month-old mice after three months of ENR housing. Data related to Fig. 1.

\section{Supplementary Data 2}

Results of GO and pathway enrichment analyses with gene targets of ENR-induced dmCpGs in 4.5 months old mice. Listed are significantly enriched pathways of "Reactome" pathway enrichment analysis (sheet 1), significantly enriched GO terms from GO cellular component enrichment analysis (sheet 2), significantly enriched GO terms of SynGO biological process enrichment analysis (sheet 3 ) and differentially methylated genes with functional annotation in MANGO (sheet 4) and all 373 differentially methylated genes (sheet 5). Data related to Fig. 1.

\section{Supplementary Data 3}

Lists of age-related differentially methylated cytosines in STD mice (sheet 1) and ENRinduced differentially methylated cytosines in aged mice (sheet 2). Data related to Fig. 2 and Supplementary Fig. 1.

\section{Supplementary Data 4}

Significantly enriched pathways of age-related differentially methylated genes from "Reactome" pathway enrichment analysis. Data related to Supplementary Fig. 2.

\section{Supplementary Data 5}

Results of transcription factor motif enrichment analysis of age-related dmCpGs counteracted by ENR. Listed are significantly enriched transcription factor motifs in all 13,314 dmCpGs (sheet 1), enriched motifs in dmCpGs located within enhancers (sheet 2) and locations and methylation changes of Mecp2 target dmCpGs (sheet 3). Data related to Fig. 3.

\section{Supplementary Data 6}

Results of enrichment analysis used for generation of the enrichment map (sheet 1) and list of the 676 genes at which ENR counteracted age-related DNA methylation changes (sheet 2).

Data related to Fig. 4.

\section{Supplementary Data 7}

List of genes that are dysregulated with age-related cognitive decline in humans and regulated by ENR in mice (sheet 1) and results of STRING enrichment analysis (sheet 2). Data related to Fig. 5. 